\title{
Fixed-Time Fuzzy Adaptive Fault-Tolerant Control for Hypersonic Flight Vehicles Using a New Prescribed Performance Function
}

\section{Zehong Dong ( $\sim$ dongzehong1995@163.com )}

Air Force Engineering University https://orcid.org/0000-0002-1838-057X

\section{Yinghui Li}

Air Force Engineering University

\section{Maolong Lv}

Delft University of Technology: Technische Universiteit Delft

Ju Park

Yeungnam University

\section{Dingshan Sun}

Delft University of Technology: Technische Universiteit Delft

\section{Research Article}

Keywords: Fixed-Time Tracking Control, Hypersonic Flight Vehicles, Actuator Failure, Prescribed Performance Control

Posted Date: March 2nd, 2021

DOI: https://doi.org/10.21203/rs.3.rs-211702/v1

License: (c) (i) This work is licensed under a Creative Commons Attribution 4.0 International License. Read Full License 
Noname manuscript No.

(will be inserted by the editor)

\section{Fixed-Time Fuzzy Adaptive Fault-Tolerant Control for Hypersonic Flight Vehicles Using a New Prescribed Performance Function}

\author{
Zehong Dong \\ Yinghui Li \\ Maolong Lv \\ Ju H. Park \\ Dingshan Sun
}

Received: date / Accepted: date

\begin{abstract}
This article proposes a new fixed-time fuzzy adaptive fault-tolerant control methodology for the longitudinal dynamics of hypersonic flight vehicles (HFVs) in the presence of actuator faults, uncertain dynamic$\mathrm{s}$ and external disturbances. In contrast with the conventional fixed-time control schemes that typically contain the fractional powers of errors in their designs, this work develops a low-complexity control structure in the sense of removing the dependence on the need of above-mentioned fractional power terms by means of prescribed performance control (PPC) method. Different from the most existing PPC approaches where the initial conditions of tracking errors are required to be known, the newly proposed prescribed performance function (PPF) can relax such restrictions through choosing properly small initial values of PPF. Fuzzy logic systems (FLSs) are employed to handle unknown dynamics and minimal learning parameter (MLP) technique is incorporated into the design for the purpose of alleviating computation burden. Closed-loop stabil-
\end{abstract}

Z. Dong

Graduate College, Air Force Engineering University, Xi'an 710038, China

Y. Li ( $\triangle)$

Aeronautics Engineering College, Air Force Engineering University, Xi'an 710038, China

E-mail: liyinghui66@163.com

M. Lv · D. Sun

Delft Center for Systems and Control, Delft University of Technology, Mekelweg 2, Delft 2628CD, The Netherlands

J. H. Park

Department of Electrical Engineering, Yeungnam University, Kyongsan 38541, South Korea ity is rigorously proved via Lyapunov stability theory and simulation results are eventually given to validate the effectiveness of the proposed control strategy.

Keywords Fixed-Time Tracking Control · Hypersonic Flight Vehicles · Actuator Failure · Prescribed Performance Control

\section{Introduction}

Hypersonic flight vehicles have already attracted considerable attention due to their advantages of high flight speed, remarkable penetration ability and cost-effectiveness [1]-[3]. Nevertheless, the controller design for HFVs remains an intractable issue due to their peculiar features. For example, the engine-airframe structure results in strong couplings between propulsive and aerodynamic forces, and there exist intricate flexible deformation due to the slender geometry of vehicle structure, which influences the aerodynamic characteristics prominently [4]. In addition, the fast time-varying flight environmen$\mathrm{t}$ as well as the unknown external disturbances lead to frequent parameter variations and model uncertainties, dramatically increasing the difficulty of controller design for HFVs [5]. To address these problems, many effective methods have been presented, including robust control [4]-[6], neural/fuzzy control [7]-[9], prescribed performance control [10]-[12] and so on. Although these efforts solve the above-mentioned issues to some extent, these results rarely focus on the rate of convergence.

To be specific, only the exponential convergence of tracking error is guaranteed in the aforementioned work, which reveals the convergence time tends to be infinite. From a practical perspective, the rate of convergence is of great significance to the transient tracking performance [13]. Recently, the finite-time tracking control for HFVs is investigated in [13]-[16], which can make the tracking error converge into the predefined compact set within a finite time. Nevertheless, the convergence time, which is commonly achieved in [13]-[16], depends on the initial states of the system. It inevitably brings up a problem, that is, the convergence time can not be accurately settled when the initial states of the system are unknown. To solve such problem, the fixed-time control [17]-[20] is proposed skillfully, by which the tracking error can converge into a predefined impact set within a fixed time and the connection between the convergence time and initial states is eliminated.

However, there still exist some shortcomings with the conventional fixed-time tracking control scheme [17][20], where the inequation $\dot{V}(x) \leq-\tau V^{p}(x)-\varsigma V^{q}(x)+$ $\kappa$ holds. On the one hand, the derivative of virtual and actual control laws will tend to infinite when the track- 
ing error approaches to zero, giving rise to singularity issues in the control design [20]. On the other hand, when the system encounters the unknown external disturbances [21]-[24] or the system faults [25]-[29], it is complicated to both ensure the fixed-time stability of the system and predetermine the convergence accuracy by selecting the design parameters. Furthermore, it is well known that the transient and steady-state performances are important to the controller design for HFVs [30]. Nonetheless, the existing PPC for HFVs commonly fails to explicitly contain a convergence time $T$ in the performance function. Thus, it is urgent to develop a new low-complexity fixed-time fault-tolerant control (FTFTC) strategy for HFVs with the prescribed performance.

Motivated by these observations, we present a fixedtime adaptive fuzzy fault-tolerant control scheme for $\mathrm{H}-$ FVs by utilizing a new prescribed performance function. The contributions mainly contain the follows:

1. This paper presents a structurally inexpensive FTFTC framework for HFVs in the sense that no fractional powers are involved in the design. Specifically, conventional fixed-time controllers in [18]-[20] need to satisfy the condition $\dot{V}(x) \leq-\tau V^{p}(x)-$ $\varsigma V^{q}(x)+\kappa$. Nevertheless, merely the form $\dot{V}(x) \leq$ $-\tau V(x)+\kappa$ is required in this work, which not only simplifies the control law but also removes the singularity problem caused by the derivative of fraction term.

2. As opposed to the state-of-the-art PPC methods [11]-[12] and [22], we propose a new PPC that not only enables us to obtain fixed-time convergence, but also removes the reliance on precise initial error values. This is done in combination with a newly proposed PPF.

3. By constructing the intermediate control laws and adaptive laws, the adverse impact of actuator faults of HFVs (e.g. loss of effectiveness, drift, etc.) is compensated effectively.

The remainder of the work is organized as follows. The HFV dynamics and preliminaries are introduced in Section 2. In Section 3, the FLSs-approximator-based FTFTC is designed and the closed-loop stability is verified in Section 4. Section 5 provides simulations to demonstrate the effectiveness of the proposed methods and the work is concluded in Section 6 .

\section{Problem formulation and preliminaries}

\subsection{Hypersonic flight vehicle dynamics}

The longitudinal control-oriental model is originally developed by Parker [31], which can be formulated as

$$
\begin{aligned}
\dot{V} & =\frac{T \cos (\theta-\gamma)-D}{m}-g \sin \gamma, \quad \dot{h}=V \sin \gamma \\
\dot{\gamma} & =\frac{T \sin (\theta-\gamma)+L}{m V}-\frac{g \cos \gamma}{V}, \quad \dot{\theta}=Q \\
\dot{Q} & =\frac{M+\widetilde{\psi}_{1} \ddot{\eta}_{1}+\widetilde{\psi}_{2} \ddot{\eta}_{2}}{I_{y y}}
\end{aligned}
$$

$k_{1} \ddot{\eta}_{1}=-2 \zeta_{1} \omega_{1} \dot{\eta}_{1}-\omega_{1}^{2} \eta_{1}+N_{1}-\frac{\widetilde{\psi}_{1} M}{I_{y y}}-\frac{\widetilde{\psi}_{1} \widetilde{\psi}_{2} \ddot{\eta}_{2}}{I_{y y}}$,

$k_{2} \ddot{\eta}_{2}=-2 \zeta_{2} \omega_{2} \dot{\eta}_{2}-\omega_{2}^{2} \eta_{2}+N_{2}-\frac{\widetilde{\psi}_{2} M}{I_{y y}}-\frac{\widetilde{\psi}_{1} \widetilde{\psi}_{2} \ddot{\eta}_{1}}{I_{y y}}$

where $T, D, L, M, N_{1}$ and $N_{2}$ are defined as

$$
\begin{aligned}
T= & \beta_{1}(h, \bar{q}) \delta_{\Phi} \alpha^{3}+\beta_{2}(h, \bar{q}) \alpha^{3}+\beta_{3}(h, \bar{q}) \delta_{\Phi} \alpha^{2}+\beta_{4}(h, \bar{q}) \alpha^{2} \\
& +\beta_{5}(h, \bar{q}) \delta_{\Phi} \alpha+\beta_{6}(h, \bar{q}) \alpha+\beta_{7}(h, \bar{q}) \delta_{\Phi}+\beta_{8}(h, \bar{q}), \\
D= & \bar{q} S C_{D}^{\alpha^{2}} \alpha^{2}+\bar{q} S C_{D}^{\alpha} \alpha+\bar{q} S C_{D}^{\delta_{e}^{2}} \delta_{e}^{2}+\bar{q} S C_{D}^{\delta_{e}} \delta_{e}+\bar{q} S C_{D}^{0}, \\
M= & z_{T} T+\bar{q} S \bar{c} C_{M, \alpha}^{\alpha^{2}} \alpha^{2}+\bar{q} S \bar{c} C_{M, \alpha}^{\alpha} \alpha+\bar{q} S \bar{c} C_{M, \alpha}^{0}+\bar{q} S \bar{c} c_{e} \delta_{e}, \\
L= & \bar{q} S C_{L}^{\alpha} \alpha+\bar{q} S C_{L}^{\delta_{e}} \delta_{e}+\bar{q} S C_{L}^{0} \\
N_{1}= & N_{1}^{\alpha^{2}} \alpha^{2}+N_{1}^{\alpha} \alpha+N_{1}^{0} \\
N_{2}= & N_{2}^{\alpha^{2}} \alpha^{2}+N_{2}^{\alpha} \alpha+N_{2}^{\delta_{e}} \delta_{e}+N_{2}^{0} \\
\bar{q}= & \frac{\bar{\rho} V^{2}}{2}, \quad \bar{\rho}=\bar{\rho}_{0} \exp \left(\frac{h_{0}-h}{h_{s}}\right)
\end{aligned}
$$

and more detailed definitions can be found in [31].

\subsection{The actuator-fault model}

The actuator-fault model is developed by [26]

$v(u)=\omega u+\varepsilon$,

where $u$ and $v(u)$ denote the input and output signals of actuator, and $\omega$ and $\varepsilon$ represent the actual control effectiveness and drift distance, respectively.

Assumption 1 There exists an unknown positive constant $\bar{\varepsilon}$ such that $|\varepsilon| \leq \bar{\varepsilon}, 0<\omega \leq 1$.

Remark 1 Assumption 1 is commonly applied in FTC research to ensure the controllability of system when the actuator faults occur [26]. During flight, actuator fault inevitably occurs due to multiple factors such as aging and damage of components or screw shedding, which deteriorates the flight performance and even causes the serious flight accident in severe circumstance. Therefore, it is of great significance to consider the possible actuator faults when designing the control strategy. With different values of $\omega$ and $\varepsilon,(3)$ can be divided into the following four cases

1. $\omega=1$ and $\varepsilon=0$, representing the fault-free case; 2. $0<\underline{\omega} \leq \omega \leq \bar{\omega}<1$ and $\varepsilon=0$, where $\underline{\omega}$ and $\bar{\omega}$ are unknown positive constants, denoting partial loss of effectiveness; 


\begin{tabular}{|llllll|}
\hline$V$ & Velocity. & $h$ & Altitude. & $\bar{c}$ & Mean aerodynamic chord. \\
$\theta$ & Pitch angle. & $\gamma$ & Flight path angle. & $\eta_{i}$ & $i$-th generalized flexible coordinate. \\
$\alpha$ & Angle of attack. & $Q$ & Pitch rate. & $S$ & Reference area. \\
$T$ & Thrust. & $D$ & Drag. & $\bar{q}$ & Dynamic pressure. \\
$L$ & Lift. & $M$ & Pitching moment. & $\zeta_{i}$ & Damping ratio for flexible mode $\eta_{i}$. \\
$I_{y y}$ & Moment of inertia. & $m$ & Vehicle mass. & $C_{D}^{\delta_{e}}$ & $i$-th order coefficient of $\delta_{e}$ in $D$. \\
$\Phi$ & Fuel equivalence ratio. & $\delta_{e}$ & Elevator angular deflection. & $C_{D}^{\alpha^{i}}$ & $i$-th order coefficient of $\alpha$ in $D$. \\
$z_{T}$ & Thrust moment arm. & $N_{i}$ & $i$-th generalized force. & $C_{D}^{0}$ & Constant term in $D$. \\
$N_{i}^{0}$ & Constant term in $N_{i}$. & $N_{i}^{\delta_{e}}$ & Contribution of $\delta_{e}$ to $N_{i}$. & $N_{i}^{\alpha^{j}}$ & $j$-th order contribution of $\alpha$ to $N_{i}$. \\
$C_{L}^{0}$ & Constant term in $L$. & $C_{M, \alpha}^{0}$ & Constant term in $M$. & $C_{M, \alpha}^{\alpha^{i}}$ & $i$-th order coefficient of $\alpha$ in $M$. \\
$C_{L}^{\delta_{e}}$ & Coefficient of $\delta_{e}$ in $L$. & $C_{L}^{\alpha}$ & Coefficient of $\alpha$ in $L$. & $\omega_{i}$ & Natural frequency for $\eta_{i}$. \\
& & & & \\
\hline
\end{tabular}

3. $\omega=1$ and $\varepsilon \neq 0$, indicating the bias fault; 4. $0<\underline{\omega} \leq \omega \leq \bar{\omega}<1$ and $\varepsilon \neq 0$, where $\underline{\omega}$ and $\bar{\omega}$ are unknown positive constants, signifying that partial loss of effectiveness and bias fault occur at the same time.

\subsection{Model transformation and decomposition}

According to (1) and (2), $V$ and $h$ are mainly regulated by $\Phi$ and $\delta_{e}$, respectively. To facilitate the controller design, the HFVs dynamics are decomposed into velocity subsystem and altitude subsystem.

Inspired by [32] and [33], the velocity subsystem is considered as

$\dot{V}=f_{V}+g_{V} v(\Phi)+d_{V}$,

where $f_{V}=\frac{\bar{q} S C_{D}^{\alpha^{2}} \alpha^{2}+\bar{q} S C_{D}^{0}}{m}-g \sin \gamma+\frac{\cos \alpha}{m}\left[\beta_{2}(h, \bar{q}) \alpha^{3}+\right.$ $\left.\beta_{4}(h, \bar{q}) \alpha^{2}+\beta_{6}(h, \bar{q}) \alpha+\beta_{8}(h, \bar{q})\right], g_{V}=\frac{\cos \alpha}{m}\left[\beta_{7}(h, \bar{q})+\right.$ $\left.\beta_{3}(h, \bar{q}) \alpha^{2}+\beta_{1}(h, \bar{q}) \alpha^{3}\right] . f_{V}$ and $g_{V}$ stand for unknown functions due to the time-varying aerodynamic parameters, while $d_{V}$ represents the external disturbance on velocity.

Inspired by [34], we take the assumption $\sin (\gamma) \approx \gamma$ and $\cos (\gamma) \approx 1$, then the velocity subsystem can be considered as

$$
\begin{cases}\dot{h}=V \gamma+d_{h}, & \dot{\gamma}=f_{\gamma}+g_{\gamma} \theta+d_{\gamma}, \\ \dot{\theta}=Q, & \dot{Q}=f_{Q}+g_{Q} v\left(\delta_{e}\right)+d_{Q},\end{cases}
$$

where $f_{\gamma}=\frac{\bar{q} S\left(C_{L}^{0}-C_{L}^{\alpha} \gamma\right)+T \sin \alpha}{m V}-\frac{g}{V}, g_{\gamma}=\frac{\bar{q} S C_{L}^{\alpha}}{m V}$, $f_{Q}=z_{T} T+\frac{\bar{q} S \bar{c} C_{M, \alpha}(\alpha)}{I_{y y}}$ and $g_{Q}=\frac{\bar{q} S \bar{c} c_{e}}{I_{y y}}$. Similar to velocity subsystem, the functions $f_{\gamma}, g_{\gamma}, f_{Q}$ and $g_{Q}$ are unknown functions; $d_{h}, d_{\gamma}$ and $d_{Q}$ are the external disturbances of altitude subsystem. Along the standard ideas as [10]-[11], we assume there exist unknown positive functions $g_{V m}, g_{\gamma m}$ and $g_{Q m}$ such that $0<g_{V m} \leq g_{V}, 0<g_{\gamma m} \leq g_{\gamma}$ and $0<g_{Q m} \leq g_{Q}$.
Remark 2 In practice, it is rather difficult to know the values of functions $f_{\bullet}, g_{\bullet}, \in\{V, \gamma, Q\}$ accurately. There are mainly the following reasons: on the one hand, the aerodynamic parameters are constantly changing with the flight environment (i.e. velocity, altitude, attack of angle, etc.), where there inevitably exist measuring errors in the sensors of flight control system [1]. On the other hand, it is impossible to take all flight environment of HFVs into account in a wind tunnel, so that we have to rely on curve fitting technology to build the aerodynamic model [6]. Consequently, an exact model for HFVs is difficult to be obtained and in order to increase the robustness of flight control system, we regard $f_{\bullet}, g_{\bullet}, \in\{V, \gamma, Q\}$ as unknown functions.

Assumption 2 [35] The reference trajectory $y_{\text {ref }}(t)$, together with its $i$-order derivative $y_{\text {ref }}^{(i)}(t)$ are continuous and bounded $(i=1,2 \cdots n)$.

\subsection{A new fixed-time performance function}

Definition 1 [35] A smooth function $\rho(t)$ is called fixedtime performance function (FTPF), if the following conditions are satisfied

1. $\rho(t)>0$, i.e., $\rho(t)$ is ensured to be a positive function;

2. $\dot{\rho} \leq 0$, that is, $\rho(t)$ is monotonically decreasing;

3. $\lim _{t \rightarrow T} \rho(t)=\rho(T)$ and $\rho(t)=\rho(T)$ for any $t>$ $T$, where $\rho(T)$ and $T$ denote an arbitrarily small positive constant and settling time, respectively.

According to Definition 1, we construct an FTPF in the form of

$\rho(t)=\left\{\begin{array}{l}\operatorname{coth}\left(\vartheta \frac{t}{T-t}+r\right)-1+\rho(T), 0 \leq t<T \\ \rho(T), t \geq T .\end{array}\right.$

Proof In view of (6), it can be derived that 


$$
\begin{aligned}
\rho\left(T^{-}\right) & =\lim _{t \rightarrow T^{-}}\left[\operatorname{coth}\left(\vartheta \frac{t}{T-t}+r\right)-1+\rho(T)\right] \\
& =\lim _{t \rightarrow T^{-}} \frac{e^{\vartheta \frac{t}{T-t}}+r}{e^{\vartheta \frac{t}{T-t}}+r}-e^{-\vartheta \frac{t}{T-t}-r} \\
& =\rho(T)=\rho\left(T^{+}\right),
\end{aligned}
$$

that is, $\rho(t)$ is a continuous function. Furthermore, we can deduce that

$$
\begin{aligned}
\dot{\rho}(t) & =-\frac{\vartheta}{T}\left(\frac{T}{T-t}\right)^{2} \operatorname{csch}^{2}\left(\vartheta \frac{t}{T-t}+r\right) \\
& =-\frac{\vartheta}{T}\left(\frac{T}{T-t}\right)^{2}\left(\frac{2}{e^{\vartheta \frac{t}{T-t}+r}-e^{-\vartheta \frac{t}{T-t}-r}}\right)^{2}
\end{aligned}
$$

when $t<T$, and $\dot{\rho}(t)=0$ when $t \geq T$. For the sake of simplification, we denote $x=\frac{t}{T-t}$ and the fact $\lim _{t \rightarrow T^{-}} x=+\infty$ holds. Then, we can obtain

$$
\lim _{t \rightarrow T^{-}} \dot{\rho}(t)=\lim _{x \rightarrow+\infty}-\frac{4 \vartheta}{T}\left(\frac{1+x}{e^{\vartheta x+r}-e^{-\vartheta x-r}}\right)^{2} .
$$

With the help of L'Hospital's rule, we get that

$\lim _{t \rightarrow T^{-}} \dot{\rho}(t)=\lim _{x \rightarrow+\infty}-\frac{4}{T \vartheta}\left(\frac{1}{e^{\vartheta x+r}-e^{-\vartheta x-r}}\right)^{2}=\dot{\rho}\left(T^{+}\right)$.

Next, $\frac{\mathrm{d} \rho^{2}(t)}{\mathrm{d} t^{2}}$ can be derived as

$$
\begin{aligned}
& \frac{\mathrm{d} \rho^{2}(t)}{\mathrm{d} t^{2}}=-\frac{8 \vartheta(1+x)}{T\left(e^{\vartheta x+r}-e^{-\vartheta x-r}\right)^{2}}-\frac{8 \vartheta^{2}(1+x)^{2}\left(e^{\vartheta x+r}+e^{-\vartheta x-r}\right)}{T\left(e^{\vartheta x+r}-e^{-\vartheta x-r}\right)^{3}} \\
& =\sqrt{|\dot{\rho}(t)|} \frac{4 \sqrt{\vartheta / T}}{e^{\vartheta x+r}-e^{-\vartheta x-r}}+\frac{T}{4} \dot{\rho}(t)\left(1+\frac{2 e^{-\vartheta x-r}}{e^{\vartheta x+r}-e^{-\vartheta x-r}}\right) .
\end{aligned}
$$

Then, we have $\lim _{t \rightarrow T^{-}} \frac{\mathrm{d} \rho^{2}(t)}{\mathrm{d} t^{2}}=\frac{\mathrm{d} \rho^{2}\left(T^{+}\right)}{\mathrm{d} t^{2}}=0$. Similarly, it leads to that $\lim _{t \rightarrow T^{-}} \frac{\mathrm{d} \rho^{i}(t)}{\mathrm{d} t^{i}}=\frac{\mathrm{d} \rho^{i}\left(T^{+}\right)}{\mathrm{d} t^{i}}=0, i=3, \ldots, n$. That is to say, $\rho(t)$ is a smooth function. Furthermore, we can also see that $\dot{\rho}(t) \leq 0$ and the function $\rho(t)$ is continuous at $T$. Thus, we can conclude that the function $\rho(t)$ is a FTPF. This completes the proof.

Remark 3 We can easily obtain sufficiently large $\rho(0)$ by selecting a sufficiently small $r$, that is to say, the initial error need not to be known accurately. Consequently, the FTPF without initial error constraint is achieved. Furthermore, we can also conclude that, the convergence rate of the error depends on $\vartheta$, which can be seen in Fig. 1 (a). By setting the steady-state error boundary as $\rho(T)=1$ and choosing different values of $\vartheta$, we can obtain that a larger $\vartheta$ means a faster convergence rate of the error.

Consider the following transformation

$q(t)=\frac{e(t)}{\rho(t)}$, where the error transformation function is chosen as

$z(q)=\frac{q}{1-q^{2}}$,

and we abbreviate $q=q(t)$ there-in-after.

Remark 4 From (8), it can be observed that the inequation $-1<q<1$ holds if $z(q)$ is bounded. In view of (7), we conclude that $|e(t)|<|\rho(t)|$ holds as long as $|e(0)|<|\rho(0)|$. Choosing $\vartheta=0.5, r=0.1, T=6$ and $\rho(T)=1$, the convergence performance of the proposed FTPF is shown in Fig. 1 (b). In contrast to traditional PPC [10], where the prescribed performance function is in the form of $\rho(t)=\operatorname{coth}(\vartheta t+r)-1+\rho_{\infty}$, the proposed control scheme explicitly contains a convergence time $T$ in the FTPF. By this means, we can easily preset the convergence time as needed.
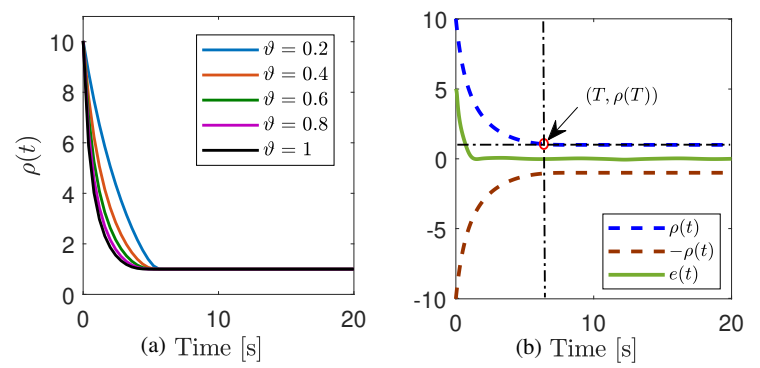

Fig. 1: The convergence performance of the proposed FTPF

\subsection{Fuzzy logic system}

Define a set of fuzzy IF-THEN rules, where the $l$ th IF-THEN rule is written as [21]-[22]

$\mathcal{R}^{l}$ : If $x_{1}$ is $F_{1}^{l}$, and $\ldots$ and $x_{n}$ is $F_{n}^{l}$, then $y$ is $B^{l}$.

where $\boldsymbol{x}=\left[x_{1}, \ldots, x_{n}\right]^{\mathrm{T}} \in \mathbb{R}^{n}$, and $y \in \mathbb{R}$ are the input and output of the FLSs respectively, and $F_{1}^{l}, \ldots, F_{n}^{l}$ and $B^{l}$ are fuzzy sets in $\mathbb{R}$. Let $F(\boldsymbol{x})$ be a continuous function defined on a compact set $\Omega_{\boldsymbol{x}}$. Then, for a given desired level of accuracy $\varepsilon>0$, there exists a FLS $\boldsymbol{W}^{\mathrm{T}} \boldsymbol{S}(\boldsymbol{x})$ such that $\sup _{\boldsymbol{x} \in \Omega_{\boldsymbol{x}}}\left|F(\boldsymbol{x})-\boldsymbol{W}^{\mathrm{T}} \boldsymbol{S}(\boldsymbol{x})\right| \leq \phi$, where $\boldsymbol{W}=\left[w_{1}, \ldots, w_{p}\right]^{\mathrm{T}}$ is the adaptive fuzzy parameter vector in a compact set $\Omega_{\boldsymbol{W}}, p$ is the number of the fuzzy rules, and $\boldsymbol{S}(\boldsymbol{x})=\left[S_{1}(\boldsymbol{x}), \ldots, S_{p}(\boldsymbol{x})\right]^{\mathrm{T}}$ is the fuzzy basis function vector with $S_{l}(\boldsymbol{x})=\prod_{j=1}^{m} \mu_{F_{j}^{l}}\left(x_{j}\right) / \sum_{l=1}^{p}\left(\prod_{j=1}^{m}\right.$ $\left.\mu_{F_{j}^{l}}\left(x_{j}\right)\right)$ where $\mu_{F_{j}^{l}}\left(x_{j}\right)$ is a fuzzy membership function of the variable $x_{j}$ in IF-THEN rule. Let $\boldsymbol{W}^{*}$ be the optimal parameter vector, which is defined as

$$
\boldsymbol{W}^{*}=\arg \min _{\boldsymbol{W} \in \Omega_{\boldsymbol{W}}}\left\{\sup _{\boldsymbol{x} \in \Omega_{\boldsymbol{x}}}\left|F(\boldsymbol{x})-\boldsymbol{W}^{\mathrm{T}} \boldsymbol{S}(\boldsymbol{x})\right|\right\} \text {. }
$$


Then, we can further obtain

$$
F(\boldsymbol{x})=\boldsymbol{W}^{* \mathrm{~T}} \boldsymbol{S}(\boldsymbol{x})+\phi,
$$

where $\phi$ is the minimum fuzzy approximation error.

Lemma 1 [36] The hyperbolic tangent function tanh $(\bullet)$ is continuous and differentiable, for $\forall \varsigma \in R$ and $\forall \mu>0$, it has

$0 \leq|\varsigma|-\varsigma \tanh \left(\frac{\varsigma}{\mu}\right) \leq 0.2785 \mu$.

Lemma 2 [37] For any positive constants $\omega$ and $\delta$, the following inequation holds

$0 \leq|\omega|-\frac{\omega^{2}}{\sqrt{\omega^{2}+\delta^{2}}} \leq \delta$

The control objective of this article is to design an fuzzy adaptive tracking controller such that

1. The velocity and altitude tracking errors are guaranteed to obey the prescribed performance boundaries at all times, and finally converge into the predefined residual sets within preassigned time $T$ even in the presentence of actuator faults;

2. All signals of the closed-loop system remain bounded.

\section{The FTFTC design}

Corresponding to the decomposition in Sect. II.C, the control design is also decomposed into a velocity control design and an altitude control design.

\subsection{Velocity controller design}

We first define the velocity tracking error as

$e_{V}=V-V_{\text {ref }}$,

where $V_{\text {ref }}$ is the velocity reference trajectory. In view of (4), (7) and (8), the time derivative of $e_{V}$ is

$\dot{e}_{V}=f_{V}+g_{V} \Phi+d_{V}-\dot{V}_{r e f}=\dot{\rho}(t) q+\dot{z}_{V} \rho(t) \frac{\partial q}{\partial z}$.

Then $\dot{z}_{V}$ can be rewritten as

$$
\begin{aligned}
\dot{z}_{V} & =\frac{f_{V}+g_{V} v(\Phi)+d_{V}-\dot{V}_{r e f}-\dot{\rho}(t) q}{\rho(t) \frac{\partial q}{\partial z}} \\
& =\Upsilon_{V}+E_{V}\left(f_{V}+g_{V}(\Phi)+d_{V}-\dot{V}_{r e f}\right),
\end{aligned}
$$

with $\Upsilon_{V}=-\dot{\rho}(t) q E_{V}, E_{V}=\frac{1}{\rho(t) \frac{\partial q}{\partial z}}$.

It can be deduced from (8) that $\frac{\partial q}{\partial z}=\frac{\left(1-q^{2}\right)^{2}}{1+q^{2}} \leq 1$ when $-1<q<1$, and noticing the fact $g_{V} \geq g_{V m}>0$ leads to
$E_{V} g_{V}=\frac{g_{V}}{\rho(t) \frac{\partial q}{\partial z}} \geq \frac{g_{V m}}{\rho(0)}=\widetilde{g}_{V m}>0$.

Choose the following quadratic function

$L_{V}=\frac{1}{2} z_{V}^{2}+\frac{1}{2 \mu_{V}} \widetilde{g}_{V m} \widetilde{\ell}_{V}^{2}$,

with $\widetilde{\ell}_{V}=\ell_{V}-\widehat{\ell}_{V}$, in which $\widehat{\ell}_{V}$ denotes the estimation of adaptive parameter $\ell_{V}$, and $\mu_{V}$ is the positive userdefined parameter.

Utilizing (3) and (14), the time derivative of $L_{V}$ is

$$
\begin{aligned}
\dot{L}_{V}= & z_{V}\left(\Upsilon_{V}+E_{V} f_{V}+E_{V} g_{V} \omega_{V} \Phi+E_{V} g_{V} \varepsilon_{V}\right. \\
& \left.+E_{V} d_{V}-E_{V} \dot{V}_{r e f}\right)-\frac{1}{\mu_{V}} \widetilde{g}_{V m} \widetilde{\ell}_{V} \dot{\hat{\ell}}_{V}
\end{aligned}
$$

Define the nonlinear function

$$
F_{V}=\Upsilon_{V}+E_{V} f_{V}+E_{V} d_{V}-E_{V} \dot{V}_{r e f}+\frac{1}{2} z_{V},
$$

where $f_{V}$ and $d_{V}$ are unknown due to the existence of unknown external disturbances and fast time-varying flight environment. A FLSs-approximator is constructed to estimate $F_{V}$ as

$F_{V}=\boldsymbol{W}_{V}^{\mathrm{T}} \boldsymbol{S}_{V}\left(\boldsymbol{x}_{V}\right)+\phi_{V}$

where $\boldsymbol{x}_{V}=\left[V, V_{\text {ref }}, \dot{V}_{r e f}\right]^{\mathrm{T}}$. Substituting (19) and (20) into (18), we have

$$
\begin{aligned}
\dot{L}_{V}= & z_{V}\left(\boldsymbol{W}_{V}^{\mathrm{T}} \boldsymbol{S}_{V}+\phi_{V}+E_{V} g_{V} \omega_{V} \Phi+E_{V} g_{V} \varepsilon_{V}\right) \\
& -\frac{1}{\mu_{V}} \widetilde{g}_{V m} \tilde{\ell}_{V} \dot{\hat{\ell}}_{V}-\frac{1}{2} z_{V}^{2} .
\end{aligned}
$$

According to Young's inequation, we can further have

$$
\begin{aligned}
z_{V} \boldsymbol{W}_{V}^{\mathrm{T}} \boldsymbol{S}_{V} & \leq \frac{z_{V}^{2}\left\|\boldsymbol{W}_{V}\right\|^{2} \boldsymbol{S}_{V}^{\mathrm{T}} \boldsymbol{S}_{V}}{4 \tau_{V}}+\tau_{V}, \\
z_{V} \phi_{V} & \leq \frac{1}{2} z_{V}^{2}+\frac{1}{2} \phi_{V}^{2}
\end{aligned}
$$

where $\tau_{V}$ is a positive constant, then we can rewrite (21) as

$$
\begin{aligned}
\dot{L}_{V} \leq & z_{V}\left(E_{V} g_{V} \omega_{V} \Phi+E_{V} g_{V} \varepsilon_{V}\right)+\frac{z_{V}^{2}\left\|\boldsymbol{W}_{V}\right\|^{2} \boldsymbol{S}_{V}^{\mathrm{T}} \boldsymbol{S}_{V}}{4 \tau_{V}} \\
& +\tau_{V}+\frac{1}{2} \phi_{V}^{2}-\frac{1}{\mu_{V}} \widetilde{g}_{V m} \widetilde{\ell}_{V} \dot{\hat{\ell}}_{V}
\end{aligned}
$$

According to Assumption 1, $\omega_{V}$ and $\varepsilon_{V}$ are unknown. Therefore, we define the upper and lower bound$\mathrm{s}$ of fault parameters to achieve robustness, which are expressed as

$$
\begin{aligned}
\underline{\omega}_{V} & =\inf \left(E_{V} g_{V} \omega_{V}\right), \vartheta_{V}=\frac{1}{\underline{\omega}_{V}}, \\
\xi_{V} & =\sup \left(E_{V} g_{V} \varepsilon_{V}\right) .
\end{aligned}
$$


Consider the Lyapunov function candidate

$L_{\bar{V}}=L_{V}+\frac{1}{2 l_{V}} \underline{\omega}_{V} \widetilde{\vartheta}_{V}^{2}+\frac{1}{2 r_{V}} \widetilde{\xi}_{V}^{2}$,

where $l_{V}>0$ and $r_{V}>0$ are the parameters to be designed, $\widetilde{\vartheta}_{V}=\vartheta_{V}-\widehat{\vartheta}_{V}$ and $\widetilde{\xi}_{V}=\xi_{V}-\widehat{\xi}_{V}$ represent estimation errors with $\widehat{\vartheta}_{V}$ and $\widehat{\xi}_{V}$ being the estimations of $\vartheta_{V}$ as $\xi_{V}$, respectively.

The time derivative of $L_{\bar{V}}$ gives

$\dot{L}_{\bar{V}}=\dot{L}_{V}-\frac{1}{l_{V}} \underline{\omega}_{V} \widetilde{\vartheta}_{V} \dot{\hat{\vartheta}}_{V}-\frac{1}{r_{V}} \widetilde{\xi}_{V} \dot{\widehat{\xi}}_{V} \cdot$

Define $\ell_{V}=\frac{\left\|\boldsymbol{W}_{V}\right\|^{2}}{\widetilde{g}_{V m}}$ and choose the intermediate control law as

$\bar{\Phi}=k_{V} z_{V}+\frac{z_{V} \widehat{\ell}_{V} \boldsymbol{S}_{V}^{\mathrm{T}} \boldsymbol{S}_{V}}{4 \tau_{V}}+\widehat{\xi}_{V} \tanh \left(\frac{z_{V}}{a_{V}}\right)$

It can be induced that

$$
\begin{aligned}
\dot{L}_{\bar{V}} \leq & z_{V} E_{V} g_{V} \omega_{V} \Phi+\frac{z_{V}^{2} \ell_{V} \widetilde{g}_{V m} \boldsymbol{S}_{V}^{\mathrm{T}} \boldsymbol{S}_{V}}{4 \tau_{V}}+\tau_{V}+\frac{1}{2} \phi_{V}^{2} \\
& -\frac{\underline{\omega}_{V}}{l_{V}} \widetilde{\vartheta}_{V} \dot{\hat{\vartheta}}_{V}+z_{V} \bar{\Phi}-k_{V} z_{V}^{2}-\frac{z_{V}^{2} \widehat{\ell}_{V} \widetilde{g}_{V m} \boldsymbol{S}_{V}^{\mathrm{T}} \boldsymbol{S}_{V}}{4 \tau_{V}} \\
& +\xi_{V}\left(\left|z_{V}\right|-z_{V} \tanh \left(\frac{z_{V}}{a_{V}}\right)\right)-\frac{\widetilde{g}_{V m}}{\mu_{V}} \tilde{\ell}_{V} \dot{\widehat{\ell}}_{V} \\
& +\frac{1}{r_{V}} \widetilde{\xi}_{V}\left(r_{V} z_{V} \tanh \left(\frac{z_{V}}{a_{V}}\right)-\dot{\widehat{\xi}}_{V}\right) .
\end{aligned}
$$

Choose the adaptive laws as follows

$\dot{\hat{\ell}}_{V}=\frac{\mu_{V} z_{V}^{2} \boldsymbol{S}_{V}^{\mathrm{T}} \boldsymbol{S}_{V}}{4 \tau_{V}}-\Gamma_{V} \widehat{\ell}_{V}$

$\dot{\hat{\xi}}_{V}=r_{V} z_{V} \tanh \left(\frac{z_{V}}{a_{V}}\right)-b_{V} \widehat{\xi}_{V}$

$\dot{\hat{\vartheta}}_{V}=l_{V} z_{V} \bar{\Phi}-c_{V} \widehat{\vartheta}_{V}$

where $a_{V}>0, b_{V}>0$ and $c_{V}>0$ are the parameters to be designed.

Substituting (31)-(33) into (30) yields

$$
\begin{aligned}
\dot{L}_{\bar{V}} \leq & z_{V} E_{V} g_{V} \omega_{V} \Phi+\tau_{V}+\frac{1}{2} \phi_{V}^{2}+\frac{\Gamma_{V} \widetilde{g}_{V m}}{\mu_{V}} \widetilde{\ell}_{V} \widehat{\ell}_{V} \\
& -\underline{\omega}_{V} \widetilde{\vartheta}_{V} z_{V} \bar{\Phi}+\frac{\underline{\omega}_{V} c_{V}}{l_{V}} \widetilde{\vartheta}_{V} \widehat{\vartheta}_{V}+z_{V} \bar{\Phi}-k_{V} z_{V}^{2} \\
& +\xi_{V}\left(\left|z_{V}\right|-z_{V} \tanh \left(\frac{z_{V}}{a_{V}}\right)\right)+\frac{b_{V}}{r_{V}} \widetilde{\xi}_{V} \widehat{\xi}_{V} .
\end{aligned}
$$

Now we design the actual control law as

$\Phi=-\frac{z_{V} \widehat{\vartheta}_{V}^{2} \bar{\Phi}^{2}}{\sqrt{z_{V}^{2} \widehat{\vartheta}_{V}^{2} \bar{\Phi}^{2}+\sigma_{V}^{2}}}$, where $\sigma_{V}>0$ is a predefined constant. According to Young's inequation, one has

$$
\begin{aligned}
& \tilde{\ell}_{V} \widehat{\ell}_{V}=\left(\ell_{V}-\widetilde{\ell}_{V}\right) \widetilde{\ell}_{V} \leq-\frac{1}{2} \widetilde{\ell}_{V}^{2}+\frac{1}{2} \ell_{V}^{2}, \\
& \widetilde{\vartheta}_{V} \widehat{\vartheta}_{V}=\left(\vartheta_{V}-\widetilde{\vartheta}_{V}\right) \widetilde{\vartheta}_{V} \leq-\frac{1}{2} \widetilde{\vartheta}_{V}^{2}+\frac{1}{2} \vartheta_{V}^{2}, \\
& \widetilde{\xi}_{V} \widehat{\xi}_{V}=\left(\xi_{V}-\widetilde{\xi}_{V}\right) \widetilde{\xi}_{V} \leq-\frac{1}{2} \widetilde{\xi}_{V}^{2}+\frac{1}{2} \xi_{V}^{2} .
\end{aligned}
$$

Substituting (35)-(38) into (34) and applying Lemma 1 and Lemma 2, we can rewrite (34) as

$$
\begin{aligned}
\dot{L}_{\bar{V}} \leq & -k_{V} z_{V}^{2}-\frac{1}{2 \mu_{V}} \Gamma_{V} \widetilde{g}_{V m} \widetilde{\ell}_{V}^{2}-\frac{1}{2 l_{V}} \underline{\omega}_{V} c_{V} \widetilde{\vartheta}_{V}^{2}-\frac{1}{2 r_{V}} b_{V} \widetilde{\xi}_{V}^{2} \\
& +\frac{1}{2 \mu_{V}} \Gamma_{V} \widetilde{g}_{V m} \ell_{V}^{2}+\frac{1}{2 l_{V}} \underline{\omega}_{V} c_{V} \vartheta_{V}^{2}+\frac{1}{2 r_{V}} b_{V} \xi_{V}^{2}+\tau_{V} \\
& +\frac{1}{2} \phi_{V}^{2}+\underline{\omega}_{V} \sigma_{V}+0.2785 a_{V} \xi_{V} .
\end{aligned}
$$

3.2 Altitude controller design

In the process of altitude controller design, the backstepping methodology is adopted to deal with complex dynamics. The virtual controllers will be designed at first, and then the intermediate control law the actual control law will be constructed to counteract the impact of actuator fault. To initiate the design process, we first define the following tracking errors

$\left\{\begin{array}{l}e_{h}=h-h_{r e f}, z_{\gamma}=\gamma-\chi_{\gamma}, \\ z_{\theta}=\theta-\chi_{\theta}, \quad z_{Q}=Q-\chi_{Q},\end{array}\right.$

with $\chi_{\gamma}, \chi_{\theta}$ and $\chi_{Q}$ representing the virtual control laws.

Step 1 Similarly to velocity controller design, one reaches

$\dot{e}_{h}=V \gamma+d_{h}-\dot{h}_{r e f}=\dot{\rho}(t) q+\dot{z}_{h} \rho(t) \frac{\partial q}{\partial z}$.

Then we have

$\dot{z}_{h}=\frac{V \gamma+d_{h}-\dot{h}_{r e f}-\dot{\rho}(t) q}{\rho(t) \frac{\partial q}{\partial z}}=\Upsilon_{h}+E_{h}\left(V \gamma+d_{h}-\dot{h}_{r e f}\right)$

with

$\Upsilon_{h}=-\dot{\rho}(t) q E_{h}, E_{h}=\frac{1}{\rho(t) \frac{\partial q}{\partial z}}$.

Noting that $\frac{\partial q}{\partial z}=\frac{\left(1-q^{2}\right)^{2}}{1+q^{2}} \leq 1$ when $-1<q<1$ and the fact that $V \geq V_{m}>0$ where $V_{m}$ is minimum permissible flight velocity, yields

$E_{h} V=\frac{V}{\rho(t) \frac{\partial q}{\partial z}} \geq \frac{V_{m}}{\rho(0)}=\widetilde{V}_{m}>0$. 
Consider the following Lyapunov function candidate

$L_{h}=\frac{1}{2} z_{h}^{2}+\frac{1}{2 \mu_{h}} \widetilde{V}_{m} \widetilde{\ell}_{h}^{2}$

with $\widetilde{\ell}_{h}=\ell_{h}-\widehat{\ell}_{h}$, in which $\widehat{\ell}_{h}$ denotes the estimation of adaptive parameter $\ell_{h}, \mu_{h}$ is the positive design parameter.

The time derivative of $L_{h}$ gives

$\dot{L}_{h}=z_{h}\left(\boldsymbol{W}_{h}^{\mathrm{T}} \boldsymbol{S}_{h}+\phi_{h}+E_{h} V \gamma\right)-\frac{1}{\mu_{h}} \widetilde{V}_{m} \widetilde{\ell}_{h} \dot{\hat{\ell}}_{h}-\frac{1}{2} z_{h}^{2}$,

with $F_{h}=\Upsilon_{h}+E_{h} d_{h}-E_{h} \dot{h}_{r e f}+\frac{1}{2} z_{h}$ being approximated by FLS $\boldsymbol{W}_{h}^{\mathrm{T}} \boldsymbol{S}_{h}\left(\boldsymbol{x}_{h}\right)+\phi_{h}$, where $\boldsymbol{x}_{h}=\left[h, h_{r e f}, \dot{h}_{r e f}\right]^{\mathrm{T}}$.

Applying Young's inequation, it gives

$\begin{aligned} z_{h} \boldsymbol{W}_{h}^{\mathrm{T}} \boldsymbol{S}_{h} & \leq \frac{z_{h}^{2}\left\|\boldsymbol{W}_{h}\right\|^{2} \boldsymbol{S}_{h}^{\mathrm{T}} \boldsymbol{S}_{h}}{4 \tau_{h}}+\tau_{h}, \\ z_{h} \phi_{h} & \leq \frac{1}{2} z_{h}^{2}+\frac{1}{2} \phi_{h}^{2}\end{aligned}$

where $\tau_{h}$ is a positive constant, noting that $\gamma=z_{\gamma}+\chi_{\gamma}$ we can further rewrite (46) as

$$
\begin{aligned}
\dot{L}_{h} \leq & E_{h} V z_{h} z_{\gamma}+z_{h} E_{h} V \chi_{\gamma}+\frac{z_{h}^{2} \ell_{h} \widetilde{V}_{m} \boldsymbol{S}_{h}^{\mathrm{T}} \boldsymbol{S}_{h}}{4 \tau_{h}} \\
& +\tau_{h}+\frac{1}{2} \phi_{h}^{2}-\frac{1}{\mu_{h}} \widetilde{V}_{m} \tilde{\ell}_{h} \dot{\hat{\ell}}_{h}
\end{aligned}
$$

with the definition of $\ell_{h}=\frac{\left\|\boldsymbol{W}_{h}\right\|^{2}}{\widetilde{V}_{m}}$. Choose the virtual control law and updating law as follows

$\chi_{\gamma}=-k_{h} z_{h}-\frac{z_{h} \widehat{\ell}_{h} \boldsymbol{S}_{h}^{\mathrm{T}} \boldsymbol{S}_{h}}{4 \tau_{h}}$,

$\dot{\hat{\ell}}_{h}=\frac{\mu_{h} z_{h}^{2} \boldsymbol{S}_{h}^{\mathrm{T}} \boldsymbol{S}_{h}}{4 \tau_{h}}-\Gamma_{h} \widehat{\ell}_{h}$.

Substituting (50)-(51) into (49) yields

$\dot{L}_{h} \leq-k_{h} z_{h}^{2}+\frac{1}{\mu_{h}} \Gamma_{h} \widetilde{V}_{m} \widetilde{\ell}_{h} \widehat{\ell}_{h}+E_{h} V z_{h} z_{\gamma}+\frac{1}{2} \phi_{h}^{2}+\tau_{h}$.

Step 2 Take the Lyapunov function candidate as

$L_{\gamma}=L_{h}+\frac{1}{2} z_{\gamma}^{2}+\frac{1}{2 \mu_{\gamma}} g_{\gamma m} \widetilde{\ell}_{\gamma}^{2}$.

Similar to step 1 , by defining $\ell_{\gamma}=\frac{\left\|\boldsymbol{W}_{\gamma}\right\|^{2}}{g_{\gamma m}}$, the time derivative of $L_{\gamma}$ can be formulated as

$$
\begin{aligned}
\dot{L}_{\gamma}= & \dot{L}_{h}+z_{\gamma}\left(\boldsymbol{W}_{\gamma}^{\mathrm{T}} \boldsymbol{S}_{\gamma}+\phi_{\gamma}+g_{\gamma} \theta\right)-\frac{1}{\mu_{\gamma}} g_{\gamma m} \tilde{\ell}_{\gamma} \dot{\hat{\ell}}_{\gamma} \\
& -E_{h} V z_{h} z_{\gamma}-\frac{1}{2} z_{\gamma}^{2}
\end{aligned}
$$

with $F_{\gamma}=f_{\gamma}+E_{h} V z_{h}+d_{\gamma}-\dot{\chi}_{\gamma}+\frac{1}{2} z_{\gamma}$ being approximated by FLS $\boldsymbol{W}_{\gamma}^{\mathrm{T}} \boldsymbol{S}_{\gamma}\left(\boldsymbol{x}_{\gamma}\right)+\phi_{\gamma}$, where $\dot{\chi}_{\gamma}=\frac{\partial \chi_{\gamma}}{\partial h} \dot{h}+$ $\frac{\partial \chi_{\gamma}}{\partial \widehat{\ell}_{h}} \dot{\widehat{\ell}}_{h}+\frac{\partial \chi_{\gamma}}{\partial h_{r e f}} \dot{h}_{r e f}$ and $\boldsymbol{x}_{\gamma}=\left[h, \gamma, h_{r e f}, \dot{h}_{r e f}, h_{r e f}^{(2)}, \widehat{\ell}_{h}\right]^{\mathrm{T}}$

Design the virtual control law and adaptive law as

$$
\begin{gathered}
\chi_{\theta}=-k_{\gamma} z_{\gamma}-\frac{z_{\gamma} \widehat{\ell}_{\gamma} \boldsymbol{S}_{\gamma}^{\mathrm{T}} \boldsymbol{S}_{\gamma}}{4 \tau_{\gamma}}, \\
\dot{\hat{\ell}}_{\gamma}=\frac{\mu_{\gamma} z_{\gamma}^{2} \boldsymbol{S}_{\gamma}^{\mathrm{T}} \boldsymbol{S}_{\gamma}}{4 \tau_{\gamma}}-\Gamma_{\gamma} \widehat{\ell}_{\gamma} .
\end{gathered}
$$

Based on (52)-(56), we obtain

$$
\begin{aligned}
\dot{L}_{\gamma} \leq & -k_{h} z_{h}^{2}-k_{\gamma} z_{\gamma}^{2}+\frac{1}{\mu_{h}} \Gamma_{h} \widetilde{V}_{m} \widetilde{\ell}_{h} \widehat{\ell}_{h}+\frac{1}{\mu_{\gamma}} \Gamma_{\gamma} g_{\gamma m} \tilde{\ell}_{\gamma} \widehat{\ell}_{\gamma} \\
& +g_{\gamma} z_{\gamma} z_{\theta}+\frac{1}{2} \phi_{h}^{2}+\frac{1}{2} \phi_{\gamma}^{2}+\tau_{h}+\tau_{\gamma} .
\end{aligned}
$$

Step 3 Consider the following Lyapunov function candidate

$L_{\theta}=L_{\gamma}+\frac{1}{2} z_{\theta}^{2}+\frac{1}{2 \mu_{\theta}} \widetilde{\ell}_{\theta}^{2}$.

Defining $\ell_{\theta}=\left\|\boldsymbol{W}_{\theta}\right\|^{2}$, the time derivative of $L_{\theta}$ gives

$$
\begin{aligned}
\dot{L}_{\theta}= & \dot{L}_{\gamma}+z_{\theta}\left(\boldsymbol{W}_{\theta}^{\mathrm{T}} \boldsymbol{S}_{\theta}+\phi_{\theta}+Q\right)-\frac{1}{\mu_{\theta}} \tilde{\ell}_{\theta} \dot{\hat{\ell}}_{\theta} \\
& -g_{\gamma} z_{\gamma} z_{\theta}-\frac{1}{2} z_{\theta}^{2},
\end{aligned}
$$

with $F_{\theta}=g_{\gamma} z_{\gamma}-\dot{\chi}_{\theta}+\frac{1}{2} z_{\theta}$ being approximated by FLS $\boldsymbol{W}_{\theta}^{\mathrm{T}} \boldsymbol{S}_{\theta}\left(\boldsymbol{x}_{\theta}\right)+\phi_{\theta}$, where $\dot{\chi}_{\theta}=\sum_{x=h, \gamma} \frac{\partial \chi_{\theta}}{\partial x} \dot{x}+\sum_{x=h, \gamma} \frac{\partial \chi_{\theta}}{\partial \widehat{\hat{\ell}}_{x}} \dot{\hat{\ell}}_{x}+$ $\sum_{i=0}^{2} \frac{\partial \chi_{\theta}}{\partial h_{r e f}^{(i)}} h_{r e f}^{(i+1)}$ and $\boldsymbol{x}_{\theta}=\left[h, \gamma, \theta, h_{r e f}, \dot{h}_{r e f}, h_{r e f}^{(2)}, h_{r e f}^{(3)}\right.$, $\left.\widehat{\ell}_{h}, \widehat{\ell}_{\gamma}\right]^{\mathrm{T}}$.

Construct the virtual control law and adaptive law

$$
\begin{gathered}
\chi_{Q}=-k_{\theta} z_{\theta}-\frac{z_{\theta} \widehat{\ell}_{\theta} \boldsymbol{S}_{\theta}^{\mathrm{T}} \boldsymbol{S}_{\theta}}{4 \tau_{\theta}}, \\
\dot{\hat{\ell}}_{\theta}=\frac{\mu_{\theta} z_{\theta}^{2} \boldsymbol{S}_{\theta}^{\mathrm{T}} \boldsymbol{S}_{\theta}}{4 \tau_{\theta}}-\Gamma_{\theta} \widehat{\ell}_{\theta} .
\end{gathered}
$$

From (58)-(61), one has

$$
\begin{aligned}
\dot{L}_{\theta} \leq & -k_{h} z_{h}^{2}-k_{\gamma} z_{\gamma}^{2}-k_{\theta} z_{\theta}^{2}+\frac{\Gamma_{h} \widetilde{V}_{m}}{\mu_{h}} \tilde{\ell}_{h} \widehat{\ell}_{h}+\frac{\Gamma_{\gamma} g_{\gamma m}}{\mu_{\gamma}} \tilde{\ell}_{\gamma} \widehat{\ell}_{\gamma}+z_{\theta} z_{Q} \\
& +\frac{\Gamma_{\theta}}{\mu_{\theta}} \widetilde{\ell}_{\theta} \widehat{\ell}_{\theta}+\frac{1}{2} \phi_{h}^{2}+\frac{1}{2} \phi_{\gamma}^{2}+\frac{1}{2} \phi_{\theta}^{2}+\tau_{h}+\tau_{\gamma}+\tau_{\theta}
\end{aligned}
$$

Step 4 Choose the following Lyapunov function candidate

$L_{Q}=L_{\theta}+\frac{1}{2} z_{Q}^{2}+\frac{1}{2 \mu_{Q}} g_{Q m} \widetilde{\ell}_{Q}^{2}$. 
Constructing $\ell_{Q}=\frac{\left\|\boldsymbol{W}_{Q}\right\|^{2}}{g_{Q m}}$ and taking the derivative of $L_{Q}$ yield

$$
\begin{gathered}
\dot{L}_{Q}=\dot{L}_{\theta}+z_{Q}\left(\boldsymbol{W}_{Q}^{\mathrm{T}} \boldsymbol{S}_{Q}+\phi_{Q}+g_{Q} \omega_{Q} \delta_{e}+g_{Q} \varepsilon_{Q}\right) \\
-\frac{1}{\mu_{Q}} g_{Q m} \widetilde{\ell}_{Q} \dot{\widehat{\ell}}_{Q}-z_{\theta} z_{Q}-\frac{1}{2} z_{Q}^{2},
\end{gathered}
$$

with $F_{Q}=f_{Q}+z_{\theta}+d_{Q}-\dot{\chi}_{\theta}+\frac{1}{2} z_{Q}$ being approximated by FLS $\boldsymbol{W}_{Q}^{\mathrm{T}} \boldsymbol{S}_{Q}\left(\boldsymbol{x}_{Q}\right)+\phi_{Q}$, where $\dot{\chi}_{Q}=\sum_{x=h, \gamma, \theta} \frac{\partial \chi_{Q}}{\partial x} \dot{x}+$ $\sum_{x=h, \gamma, \theta} \frac{\partial \chi_{Q}}{\partial \widehat{\ell}_{x}} \dot{\widehat{\ell}}_{x}+\sum_{i=0}^{3} \frac{\partial \chi_{Q}}{\partial h_{r e f}^{(i)}} h_{r e f}^{(i+1)}$ and $\boldsymbol{x}_{Q}=\left[h, \gamma, \theta, Q, h_{r e f}\right.$, $\left.\dot{h}_{r e f}, h_{r e f}^{(2)}, h_{r e f}^{(3)}, h_{r e f}^{(4)}, \widehat{\ell}_{h}, \widehat{\ell}_{\gamma}, \widehat{\ell}_{\theta}\right]^{\mathrm{T}}$.

The upper and lower bounds of fault parameters are defined as

$\underline{\omega}_{Q}=\inf \left(g_{Q} \omega_{Q}\right), \vartheta_{Q}=\frac{1}{\underline{\omega}_{Q}}$,

$\xi_{Q}=\sup \left(g_{Q} \varepsilon_{Q}\right)$.

Construct the Lyapunov function

$L_{\bar{Q}}=L_{Q}+\frac{1}{2 l_{Q}} \underline{\omega}_{Q} \widetilde{\vartheta}_{Q}^{2}+\frac{1}{2 r_{Q}} \widetilde{\xi}_{Q}^{2}$,

where $l_{Q}>0$ and $r_{Q}>0$ are designed parameters, $\widetilde{\vartheta}_{Q}=\vartheta_{Q}-\widehat{\vartheta}_{Q}$ and $\widetilde{\xi}_{Q}=\xi_{Q}-\widehat{\xi}_{Q}$ represent estimation errors with $\widehat{\vartheta}_{Q}$ and $\widehat{\xi}_{Q}$ being the estimations of $\vartheta_{Q}$ and $\xi_{Q}$, respectively. Choose the intermediate control law and adaptive laws as follows

$\bar{\delta}_{e}=k_{Q} z_{Q}+\frac{z_{Q} \widehat{\ell}_{Q} \boldsymbol{S}_{Q}^{\mathrm{T}} \boldsymbol{S}_{Q}}{4 \tau_{Q}}+\widehat{\xi}_{Q} \tanh \left(\frac{z_{Q}}{a_{Q}}\right)$,

$\dot{\hat{\ell}}_{Q}=\frac{\mu_{Q} z_{Q}^{2} \boldsymbol{S}_{Q}^{\mathrm{T}} \boldsymbol{S}_{Q}}{4 \tau_{Q}}-\Gamma_{Q} \widehat{\ell}_{Q}$,

$\dot{\widehat{\hat{\xi}}}_{Q}=r_{Q} z_{Q} \tanh \left(\frac{z_{Q}}{a_{Q}}\right)-b_{Q} \widehat{\xi}_{Q}$,

$\dot{\widehat{\vartheta}}_{Q}=l_{Q} z_{Q} \bar{\delta}_{e}-c_{Q} \widehat{\vartheta}_{Q}$,

where $k_{Q}, \tau_{Q}, a_{Q}, b_{Q}$ and $c_{Q}$ are designed positive parameters.

Finally, we choose the actual control law as

$\delta_{e}=-\frac{z_{Q} \widehat{\vartheta}_{Q}^{2} \bar{\delta}_{e}^{2}}{\sqrt{z_{Q}^{2} \widehat{\vartheta}_{Q}^{2} \bar{\delta}_{e}^{2}+\sigma_{Q}^{2}}}$,

where $\sigma_{Q}>0$ is a predefined constant. Following similar analysis to velocity subsystem, we can further de- duce that

$$
\begin{aligned}
& \dot{L}_{\bar{Q}} \leq-k_{h} z_{h}^{2}-k_{\gamma} z_{\gamma}^{2}-k_{\theta} z_{\theta}^{2}-k_{Q} z_{Q}^{2}-\frac{\Gamma_{h} \widetilde{V}_{m}}{\mu_{h}} \widetilde{\ell}_{h}^{2} \\
& -\frac{\Gamma_{\gamma} g_{\gamma m}}{\mu_{\gamma}} \widetilde{\ell}_{\gamma}^{2}-\frac{\Gamma_{\theta}}{\mu_{\theta}} \widetilde{\ell}_{\theta}^{2}-\frac{\Gamma_{Q} \widetilde{g}_{Q m}}{2 \mu_{Q}} \widetilde{\ell}_{Q}^{2}-\frac{\underline{\omega}_{Q} c_{Q}}{2 l_{Q}} \widetilde{\vartheta}_{Q}^{2} \\
& -\frac{b_{Q}}{2 r_{Q}} \widetilde{\xi}_{Q}^{2}+\frac{\Gamma_{h} \widetilde{V}_{m}}{\mu_{h}} \ell_{h}^{2}+\frac{\Gamma_{\gamma} g_{\gamma m}}{\mu_{\gamma}} \ell_{\gamma}^{2}+\frac{\Gamma_{\theta}}{\mu_{\theta}} \ell_{\theta}^{2} \\
& +\frac{\Gamma_{Q} \widetilde{g}_{Q m}}{2 \mu_{Q}} \ell_{Q}^{2}+\frac{\underline{\omega}_{Q} c_{Q}}{2 l_{Q}} \vartheta_{Q}^{2}+\frac{b_{Q}}{2 r_{Q}} \xi_{Q}^{2}+\frac{1}{2} \phi_{h}^{2} \\
& +\frac{1}{2} \phi_{\gamma}^{2}+\frac{1}{2} \phi_{\theta}^{2}+\frac{1}{2} \phi_{Q}^{2}+\tau_{h}+\tau_{\gamma}+\tau_{\theta} \\
& +\tau_{Q}+\underline{\omega}_{Q} \sigma_{Q}+0.2785 a_{Q} \xi_{Q} \text {. }
\end{aligned}
$$

The whole FTFTC design for HFVs is shown in Fig.2.

\section{Closed-loop stability analysis}

Theorem 1 Despite the occurrence of unknown actuator fault (3), consider the closed-loop system composed by (4) and (5), the virtual control laws (50), (55) and (60), the intermediate control laws (29) and (68), the actual control laws (35) and (72), and the parameter adaptation laws (31)-(33), (51), (56), (61) and (69)(71). Let Assumptions 1-2 hold. By designing the parameters properly, it therefore holds that

1. The tracking errors $e_{V}$ and $e_{h}$ can converge into a predefined residual set within an user-defined time $T$.

2. The overshoot and convergence rate are guaranteed by FTPF and all signals of the closed-loop system are SGPFS.

Proof Take the Lyapunov function candidate as

$L=L_{\bar{V}}+L_{\bar{Q}}$.

Applying (39) and (73), the derivative of $L$ gives

$$
\begin{aligned}
\dot{L} \leq & -k_{V} z_{V}^{2}-k_{h} z_{h}^{2}-k_{\gamma} z_{\gamma}^{2}-k_{\theta} z_{\theta}^{2}-k_{Q} z_{Q}^{2}-\frac{\Gamma_{V} \widetilde{g}_{V m}}{2 \mu_{V}} \widetilde{\ell}_{V}^{2} \\
& -\frac{\Gamma_{h} \widetilde{V}_{m}}{\mu_{h}} \widetilde{\ell}_{h}^{2}-\frac{\Gamma_{\gamma} g_{\gamma m}}{\mu_{\gamma}} \widetilde{\ell}_{\gamma}^{2}-\frac{\Gamma_{\theta}}{\mu_{\theta}} \widetilde{\ell}_{\theta}^{2}-\frac{\Gamma_{Q} \widetilde{g}_{Q m} \widetilde{\ell}_{Q}^{2}}{2 \mu_{Q}} \\
& -\frac{\underline{\omega}_{V} c_{V}}{2 l_{V}} \widetilde{\vartheta}_{V}^{2}-\frac{\underline{\omega}_{Q} c_{Q}}{2 l_{Q}} \widetilde{\vartheta}_{Q}^{2}-\frac{b_{V}}{2 r_{V}} \widetilde{\xi}_{V}^{2}-\frac{b_{Q}}{2 r_{Q}} \widetilde{\xi}_{Q}^{2} \\
& +\frac{\Gamma_{h} \widetilde{V}_{m}}{\mu_{h}} \ell_{h}^{2}+\frac{\Gamma_{\gamma} g_{\gamma m}}{\mu_{\gamma}} \ell_{\gamma}^{2}+\frac{\Gamma_{\theta}}{\mu_{\theta}} \ell_{\theta}^{2}+\frac{\Gamma_{V} \widetilde{g}_{V m}}{2 \mu_{V}} \ell_{V}^{2}
\end{aligned}
$$




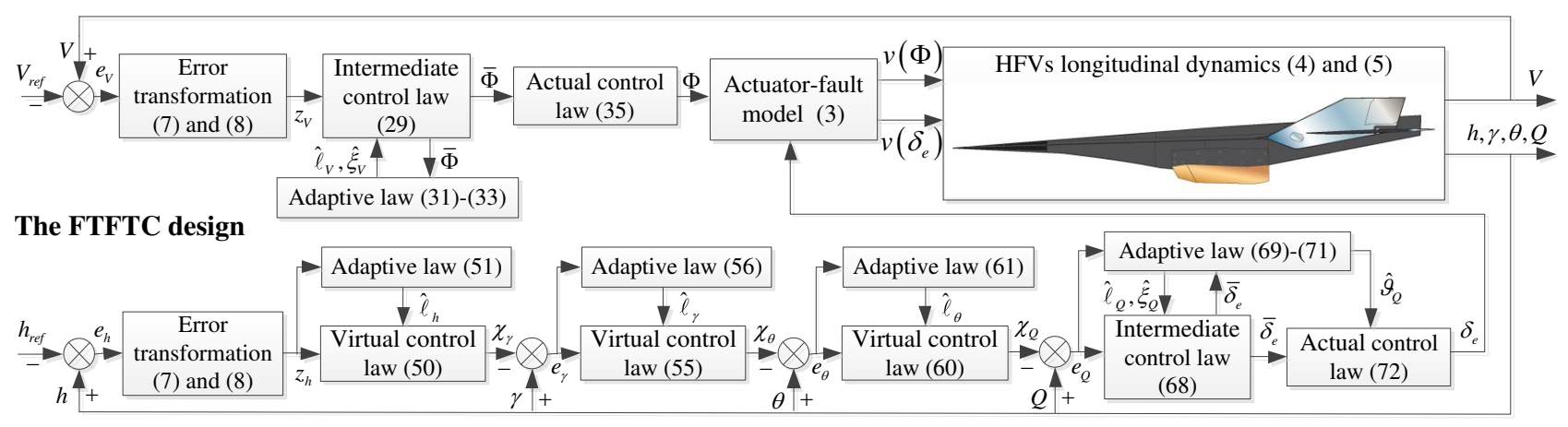

Fig. 2: Overall fixed-time tolerant-control scheme for HFVs

$$
\begin{aligned}
& +\frac{\Gamma_{Q} \widetilde{g}_{Q m}}{2 \mu_{Q}} \ell_{Q}^{2}+\frac{\underline{\omega}_{V} c_{V}}{2 l_{V}} \vartheta_{V}^{2}+\frac{\underline{\omega}_{Q} c_{Q}}{2 l_{Q}} \vartheta_{Q}^{2}+\frac{b_{V}}{2 r_{V}} \xi_{V}^{2} \\
& +\frac{b_{Q}}{2 r_{Q}} \xi_{Q}^{2}+\frac{1}{2} \phi_{V}^{2}+\frac{1}{2} \phi_{h}^{2}+\frac{1}{2} \phi_{\gamma}^{2}+\frac{1}{2} \phi_{\theta}^{2}+\frac{1}{2} \phi_{Q}^{2} \\
& +\tau_{V}+\tau_{h}+\tau_{\gamma}+\tau_{\theta}+\tau_{Q}+\underline{\omega}_{V} \sigma_{V}+\underline{\omega}_{Q} \sigma_{Q} \\
& +0.2785 a_{V} \xi_{V}+0.2785 a_{Q} \xi_{Q} .
\end{aligned}
$$

Recalling the definitions of $L_{\bar{V}}$ and $L_{\bar{Q}}$, the following inequation holds

$\dot{L} \leq-k L+C$,

where $k=\min \left\{2 k_{V}, 2 k_{h}, 2 k_{\gamma}, 2 k_{\theta}, 2 k_{Q}, \Gamma_{V}, \Gamma_{h}, \Gamma_{\gamma}, \Gamma_{\theta}\right.$, $\left.\Gamma_{Q}, b_{V}, b_{Q}, c_{V}, c_{Q}\right\}, C=\frac{\Gamma_{h} \widetilde{V}_{m}}{\mu_{h}} \ell_{h}^{2}+\frac{\Gamma_{\gamma} g_{\gamma m}}{\mu_{\gamma}} \ell_{\gamma}^{2}+\frac{\Gamma_{\theta}}{\mu_{\theta}} \ell_{\theta}^{2}+$ $\frac{\Gamma_{V} \widetilde{g}_{V m}}{2 \mu_{V}} \ell_{V}^{2}+\frac{\Gamma_{Q} \widetilde{g}_{Q m}}{2 \mu_{Q}} \ell_{Q}^{2}+\frac{\underline{\omega}_{V} c_{V}}{2 l_{V}} \vartheta_{V}^{2}+\frac{\underline{\omega}_{Q} c_{Q}}{2 l_{Q}} \vartheta_{Q}^{2}+\frac{b_{V}}{2 r_{V}} \xi_{V}^{2}+$ $\frac{b_{Q}}{2 r_{Q}} \xi_{Q}^{2}+\frac{1}{2} \phi_{V}^{2}+\frac{1}{2} \phi_{h}^{2}+\frac{1}{2} \phi_{\gamma}^{2}+\frac{1}{2} \phi_{\theta}^{2}+\frac{1}{2} \phi_{Q}^{2}+\tau_{V}+\tau_{h}+\tau_{\gamma}+$ $\tau_{\theta}+\tau_{Q}+\underline{\omega}_{V} \sigma_{V}+\underline{\omega}_{Q} \sigma_{Q}+0.2785 a_{V} \xi_{V}+0.2785 a_{Q} \xi_{Q}$.

Integrating both sides of (76) yields

$L \leq L(0)+\frac{C}{k}$.

In accordance with (17), (27), (45), (53), (58), (63), (67), (74) and (77), we have

$z_{V} \leq \sqrt{2 L(0)+\frac{C}{k}}, \quad z_{h} \leq \sqrt{2 L(0)+\frac{C}{k}}$,

and the estimation errors of the adaptive parameters will converge to the following compact sets

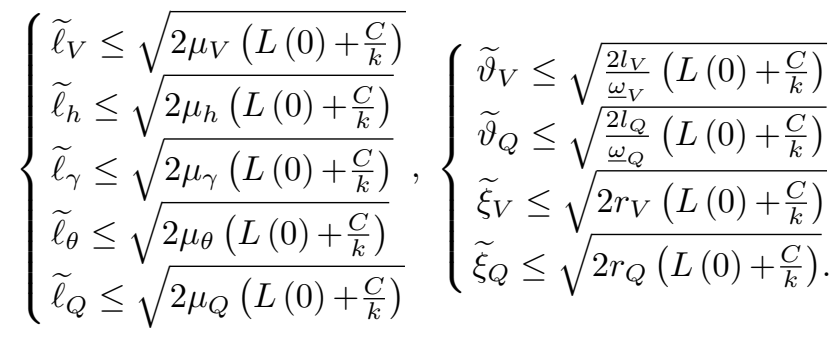

Therefore, the transformational errors $z_{V}$ and $z_{h}$ are bounded. By reviewing (6)-(8), we can conclude that the velocity and altitude tracking errors converge to a residual set within a fixed time $T$ and the prescribed performances are guaranteed. Besides, all signals of closed-loop system are SGPFS. This completes the proof.

Remark 5 The existing fixed-time control strategies for HFVs [17]-[19] fail to take system transient and steadystate performances into account and it is fairly complicated to make tracking error convergence into a preassigned compact set within the fixed-time by selecting design parameters. It is worth noting that the fixedtime tracking control is achieved as long as the bounded condition is satisfied in the proposed design. Consequently, the complexity of the control structure is reduced and the initial states need not to be known accurately via the proposed control approach.

Remark 6 It is worth mentioning that the control performance is depended closely on the designed parameters of prescribed function. In (6), large initial errors are allowed by choosing a small enough $r$, larger $\vartheta$ and smaller $T$ will increase the convergence rate. However, too large $\vartheta$ or too small $T$ will give rise to actuator input saturations. In (29), (50), (55), (60) and (68), designed parameters $k_{V}, \tau_{V}, a_{V}, k_{h}, \tau_{h}, k_{\gamma}, \tau_{\gamma}, k_{\theta}, \tau_{\theta}, k_{Q}, \tau_{Q}$ and $a_{Q}$ determine the convergence rate and convergence accuracy. In (31)-(33), (51), (56), (61) and (69)-(71), designed parameters $\mu_{V}, \Gamma_{V}, r_{V}, b_{V}, l_{V}, c_{V}, \mu_{h}, \Gamma_{h}, \mu_{\gamma}$, $\Gamma_{\gamma}, \mu_{\theta}, \Gamma_{\theta}, \mu_{Q}, \Gamma_{Q}, r_{Q}, b_{Q}, l_{Q}$ and $c_{Q}$ effect convergence rate of the adaptive parameters. In the controller design, we need to design the parameters properly to improve the tracking performance and avoid the saturation phenomenon.

\section{Simulations}

In this section, simulation results are used to demonstrate the effectiveness and superiority of the proposed methodology. The model parameters of HFVs can be consulted from [31]. HFVs are expected to climb a maneuver from the initial trim conditions, depicted in Ta- 


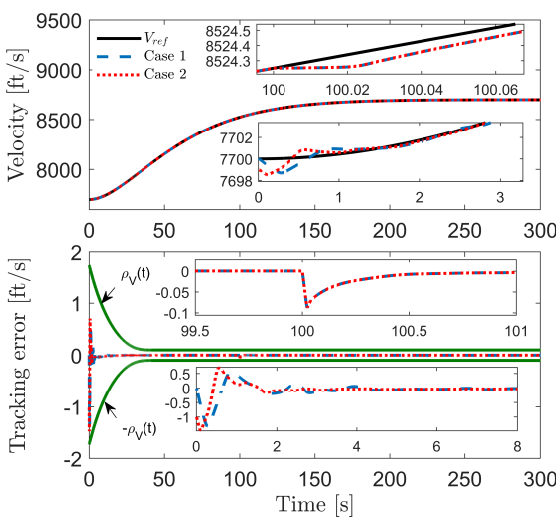

(a) Velocity tracking performance

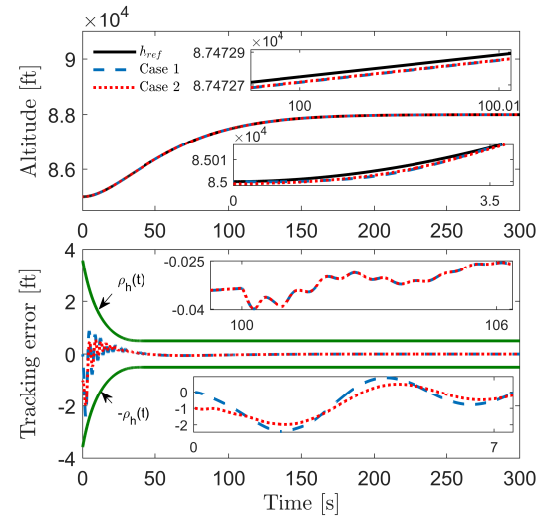

(b) Altitude tracking performance

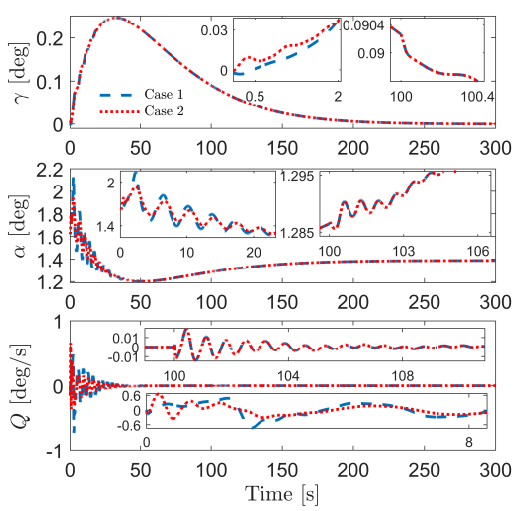

(c) Attitude angles

Fig. 3: The velocity, altitude tracking performance and attitude angles under different initial states

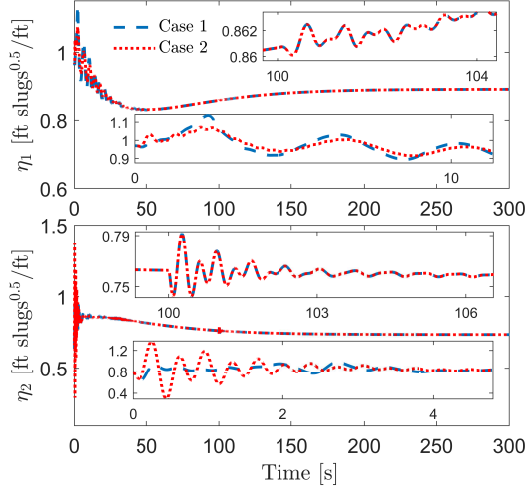

(a) Flexible states

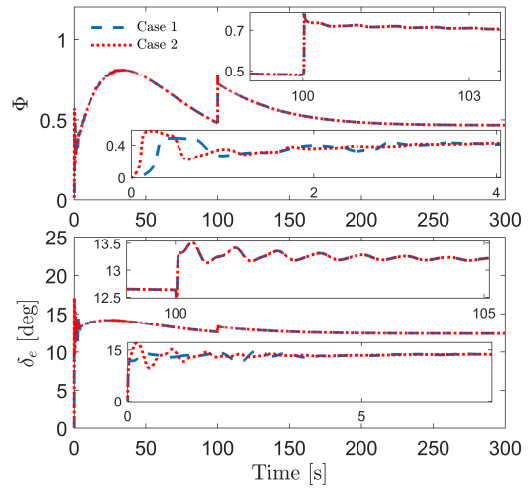

(b) Control inputs
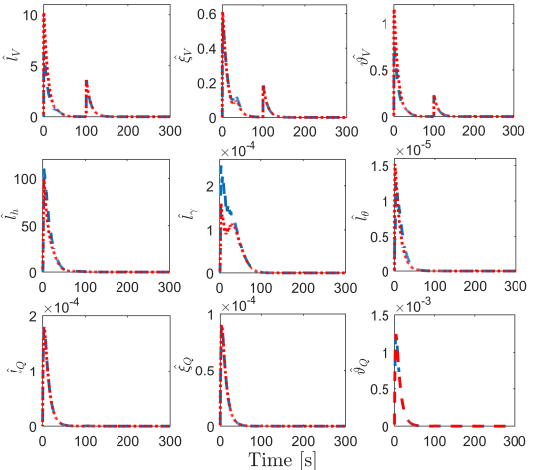

- - Case 1 - - Case 2

(c) Adaptive parameters

Fig. 4: The flexible states, control inputs and adaptive parameters under different initial states

ble 1 , to the final values $V=8700 \mathrm{ft} / \mathrm{s}$ and $h=88000 \mathrm{ft}$. The uncertain aerodynamic coefficients are modelled as $C_{i}=C_{i}^{*}\left(1+\Delta_{i}\right)$, where $C_{i}^{*}$ represents the nominal coefficient and $\Delta_{i}$ represents the uncertain factor ranging from $-20 \%$ to $20 \%$. The external disturbances in velocity subsystem and altitude subsystem are set as $d_{V}=10 \sin (t) \mathrm{ft} / \mathrm{s}, d_{h}=5 \sin (0.1 t) \mathrm{ft}, d_{\gamma}=$ $0.05 \sin (t) \mathrm{deg}, d_{Q}=0.5 \sin (0.1 t) \mathrm{deg} / \mathrm{s}$. The reference trajectories of velocity and altitude are generated via the following filters [10]

$\frac{V_{r e f}(s)}{V_{c}(s)}=\frac{0.03^{2}}{s^{2}+2 \times 0.95 \times 0.03 \times s+0.03^{2}}$,

$\frac{h_{r e f}(s)}{h_{c}(s)}=\frac{0.03^{2}}{s^{2}+2 \times 0.95 \times 0.03 \times s+0.03^{2}}$,

where $V_{\text {ref }}(s)$ and $h_{\text {ref }}(s)$ represent the inputs of filters, $V_{c}(s)$ and $h_{c}(s)$ represent the outputs of filters, respectively. It is assumed that HFVs actuators failed at $100 \mathrm{~s}$, and the details of failure are formulated in the
Table 1: The initial states

\begin{tabular}{lll}
\hline States & Value & Units \\
\hline$V$ & 7700 & $\mathrm{ft} / \mathrm{s}$ \\
$h$ & 85000 & $\mathrm{ft}$ \\
$\gamma$ & 0 & $\mathrm{deg}$ \\
$\theta$ & 1.6325 & $\mathrm{deg}$ \\
$Q$ & 0 & $\mathrm{deg} / \mathrm{s}$ \\
$\eta_{1}$ & 0.97 & $\mathrm{ft} \mathrm{slugs}{ }^{0.5} / \mathrm{ft}$ \\
$\dot{\eta}_{1}$ & 0 & $\mathrm{ft} / \mathrm{s} \mathrm{slugs}^{0.5} / \mathrm{ft}$ \\
$\eta_{2}$ & 0.7967 & $\mathrm{ft} \mathrm{slugs}{ }^{0.5} / \mathrm{ft}$ \\
$\dot{\eta}_{2}$ & 0 & $\mathrm{ft} / \mathrm{s} \mathrm{slugs}^{0.5} / \mathrm{ft}$ \\
\hline
\end{tabular}

form of

$v(\Phi)=0.8 \Phi-0.1$,

$v\left(\delta_{e}\right)=0.8 \delta_{e}+0.0349$. 


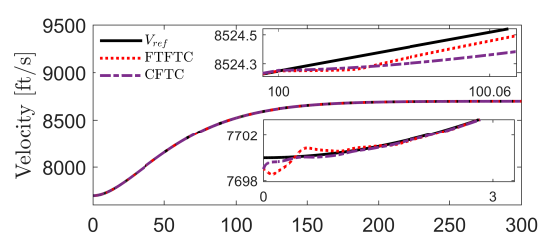

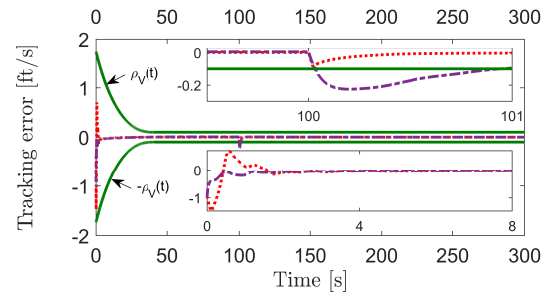

(a) Velocity tracking performance

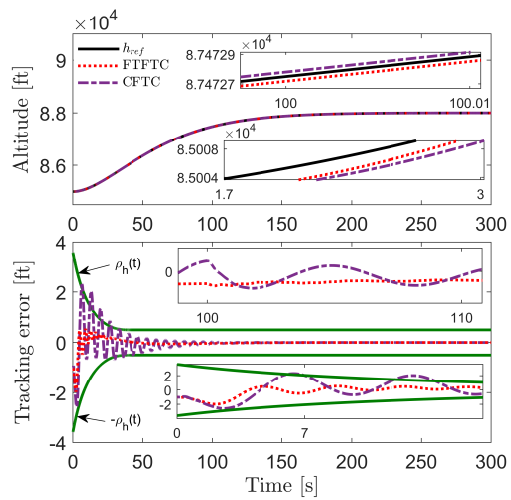

(b) Altitude tracking performance

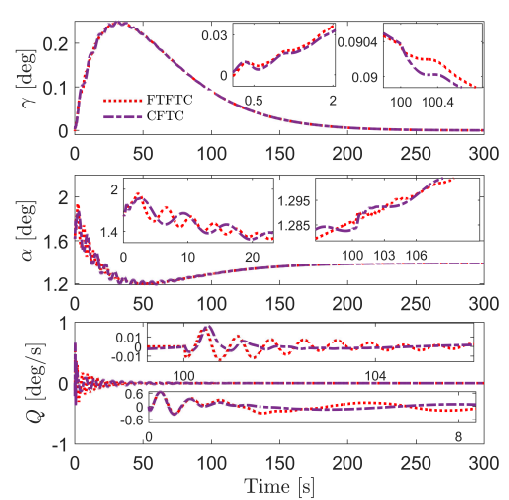

(c) Attitude angles

Fig. 5: The velocity, altitude tracking performance and attitude angles via different methodologies

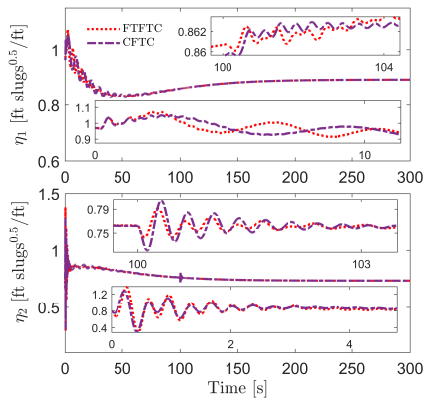

(a) Flexible states

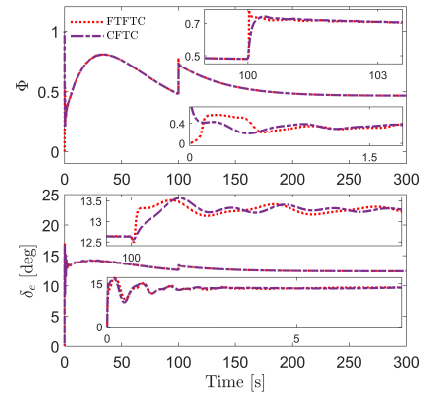

(b) Control inputs

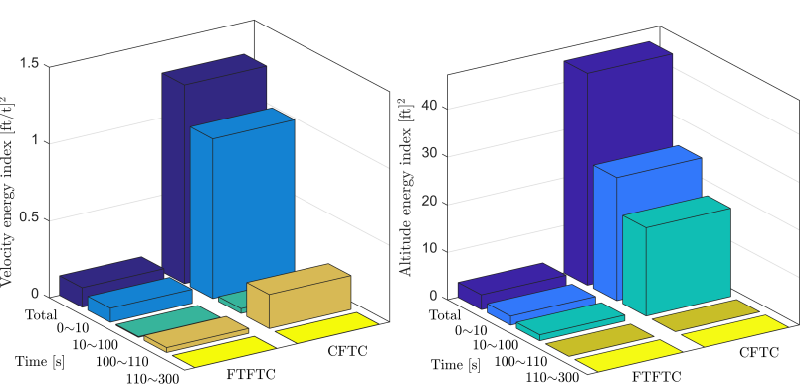

(c) Performance indexes

Fig. 6: The flexible states, control inputs and performance indexes via different methodologies

The FTPFs are selected as

$$
\begin{aligned}
\rho_{V}(t) & =\left\{\begin{array}{l}
\operatorname{coth}\left(\frac{t}{50-t}+0.4\right)-0.9,0 \leq t<50 \\
0.1, \quad t \geq 50,
\end{array}\right. \\
\rho_{h}(t) & =\left\{\begin{array}{l}
\operatorname{coth}\left(\frac{t}{50-t}+0.4\right)-0.5,0 \leq t<50 \\
0.5, \quad t \geq 50 .
\end{array}\right.
\end{aligned}
$$

The fuzzy rules in $\boldsymbol{W}_{V}^{* \mathrm{~T}} \boldsymbol{S}_{V}$ are listed as

$\mathcal{R}^{l}$ : If $V$ is $F_{V}^{i}$, then $y$ is $B^{l}$, where $i=1,2,3 ; l=$ $1,2,3$.

The fuzzy rules in $\boldsymbol{W}_{\gamma}^{* \mathrm{~T}} \boldsymbol{S}_{\gamma}$ are listed as

$\mathcal{R}^{l}$ : If $h$ is $F_{h}^{i}$, and $\gamma$ is $F_{\gamma}^{j}$, then $y$ is $B^{l}$, where $i=1,2,3 ; j=1,2,3 ; l=1,2, \ldots, 9$.

Then, the fuzzy rules in $\boldsymbol{W}_{\theta}^{* \mathrm{~T}} \boldsymbol{S}_{\theta}$ are listed as

$\mathcal{R}^{l}$ : If $h$ is $F_{h}^{i}$, and $\gamma$ is $F_{\gamma}^{j}$, and $\theta$ is $F_{\theta}^{k}$, then $y$ is $B^{l}$, where $i=1,2,3 ; j=1,2,3 ; k=1,2,3 ; l=1,2, \ldots, 27$.

The fuzzy rules in $\boldsymbol{W}_{Q}^{* \mathrm{~T}} \boldsymbol{S}_{Q}$ are listed as

$\mathcal{R}^{l}$ : If $h$ is $F_{h}^{i}$, and $\gamma$ is $F_{\gamma}^{j}$, and $\theta$ is $F_{\theta}^{k}$, and $Q$ is $F_{Q}^{p}$, then $y$ is $B^{l}$, where $i=1,2,3 ; j=1,2,3 ; k=1,2,3$; $p=1,2,3 ; l=1,2, \ldots, 81$.
The fuzzy membership function is given as follows

$\mu_{F_{V}^{1}}=\exp \left[-(V-7500)^{2} / 300\right], \quad \mu_{F_{h}^{1}}=\exp \left[-(h-85000)^{2} / 1000\right]$,

$\mu_{F_{V}^{2}}=\exp \left[-(V-8100)^{2} / 300\right], \quad \mu_{F_{h}^{2}}=\exp \left[-(h-87000)^{2} / 1000\right]$,

$\mu_{F_{V}^{3}}=\exp \left[-(V-8700)^{2} / 300\right], \quad \mu_{F_{h}^{3}}=\exp \left[-(h-89000)^{2} / 1000\right]$,

$\mu_{F_{Q}^{1}}=\exp \left[-(Q+0.03)^{2} / 0.002\right], \mu_{F_{\theta}^{1}}=\exp \left[-(\theta-0)^{2} / 0.002\right]$,

$\mu_{F_{Q}^{2}}=\exp \left[-(Q-0)^{2} / 0.002\right], \quad \mu_{F_{\theta}^{2}}=\exp \left[-(\theta-0.02)^{2} / 0.002\right]$,

$\mu_{F_{Q}^{3}}=\exp \left[-(Q-0.03)^{2} / 0.002\right], \mu_{F_{\theta}^{3}}=\exp \left[-(\theta-0.04)^{2} / 0.002\right]$,

$\mu_{F_{\gamma}^{1}}=\exp \left[-(\gamma-0)^{2} / 0.0005\right], \quad \mu_{F_{\gamma}^{2}}=\exp \left[-(\gamma-0.005)^{2} / 0.0005\right]$,

$\mu_{F_{\gamma}^{3}}=\exp \left[-(\gamma-0.01)^{2} / 0.0005\right]$.

The controller parameters are selected as $k_{V}=\mu_{V}=$ $r_{V}=l_{V}=\mu_{h}=\mu_{\gamma}=\mu_{\theta}=\mu_{Q}=r_{Q}=l_{Q}=1$, $\widetilde{g}_{V m}=0.00001, \widetilde{g}_{Q m}=1, \tau_{V}=\Gamma_{V}=c_{V}=b_{V}=\tau_{h}=$ $\Gamma_{h}=\tau_{\gamma}=\Gamma_{\gamma}=\tau_{\theta}=\Gamma_{\theta}=\tau_{Q}=\Gamma_{Q}=c_{Q}=b_{Q}=0.1$, $a_{V}=0.2, a_{Q}=0.25, \sigma_{V}=\sigma_{Q}=\frac{1}{t^{2}+0.01}, k_{h}=10$, $k_{\gamma}=2, k_{\theta}=10, k_{Q}=50$. To prove that the proposed FTFTC is realizable and independent of the initial states, the first example is taken, while the superiority 
of the proposed FTFTC over the conventional fixedtime control (CFTC) in [38] is explained in the second example.

Example 1 In this example, two different initial states cases, i.e., case $1\left(e_{V}(0)=e_{h}(0)=0\right)$ and case 2( $\left.e_{V}(0)=e_{h}(0)=1\right)$ are considered respectively.

The obtained simulation results, depicted in Figs. 34 , reveal that the proposed FTFTC has full capabilities to deal with the condition that aerodynamic parameters are perturbed and initial states are uncertain. It can be observed from Figs. 3 (a)-(b) that the velocity and altitude tracking errors are guaranteed not to exceed the prescribed bounds. Furthermore, the velocity and altitude can rapidly track their reference trajectories even if the actuator fails. Fig. 3 (c) and Fig. 4 (a) depict there is no high-frequency chattering in the attitude angles as well as the flexible states, and they can converge to their steady values rapidly. Fig. 4 (b) shows that the control inputs are smooth and within realistic limits. The estimated values of adaptive parameters are bounded, which is presented in Fig. 4 (c).

Example 2 In this example, simulations via the CFTC $[38]$ and the proposed FTFTC are demonstrated, where the initial states are set as $e_{V}(0)=e_{h}(0)=1$. In order to expound the advantages of tracking performances of the proposed FTFTC, the performance index $E=$ $\int_{0}^{t} e^{2} \mathrm{~d} t$ is introduced where $e$ denotes the tracking error.

Simulation results are depicted in Figs. 5-6. Figs. 5 (a)-(b) shows the velocity and altitude tracking performance, in which the velocity and altitude tracking errors are limited in the preset bounds by the proposed FTFTC and the proposed FTFTC can provide higher rate of convergence compared with the CFTC. Besides, the attitude angles and flexible states are shown in Fig. 5 (c) and Fig. 6 (a), indicating that smaller oscillation amplitudes of attitude angles and flexible states are achieved in the presence of actuator failures by means of the proposed FTFTC. In addition, Fig. 6 (c) gives that less error energy is produced via the proposed FTFTC in contrast with the CFTC. That is to say, the proposed FTFTC can achieve more accurate tracking.

\section{Conclusions}

A novel fixed-time fuzzy adaptive fault-tolerant control methodology based on performance function is developed for hypersonic flight vehicles in this work. In contrast with the conventional fixed-time control, the proposed approach not only guarantees that the velocity and altitude tracking errors converge into a preassigned compact set, but also satisfies both the prescribed transient and steady performance. In addition, the proposed scheme can avoid the singularity problem caused by the differential of fractional order tracking error and remain valid in spite of actuator faults. Comparative simulation results confirm the validity and superiority of the presented control strategy.

Acknowledgements This work was supported by the National Research Foundation of Korea (NRF) grant funded by the Korea government (MSIT) (No. 2020R1A2B5B02002002).

\section{Conflict of interest}

The authors declare that they have no conflict of interest.

\section{References}

1. Fiorentini, L., Serrani, A.: Adaptive restricted trajectory tracking for a non-minimum phase hypersonic vehicle model. Automatica. 48(7), 1248-1261 (2012)

2. Shen, Q., Jiang, B., Cocquempot, V.: Fault tolerant control for T-S fuzzy systems with application to near space hypersonic vehicle with actuator faults. IEEE Transactions on Fuzzy Systems. 20(4), 652-665 (2012)

3. Wu, H., Liu, Z., Guo, L.: Robust L1-gain fuzzy disturbance observer-based control design with adaptive bounding for a hypersonic vehicle. IEEE Transactions on Fuzzy Systems. 22(6), 1401-1412 (2014)

4. Cheng, X., Wang, P., Tang, G.: Fuzzy-reconstructionbased robust tracking control of an air-breathing hypersonic vehicle. Aerospace ence and Technology. 86, 694-703 (2019)

5. Cheng, Y., Xu, B., Wu, F., Hu, X., Hong, R.: HOSM observer based robust adaptive hypersonic flight control using composite learning. Neurocomputing. 295, 98-107 (2018)

6. $\mathrm{Hu}, \mathrm{X} ., \mathrm{Xu}, \mathrm{B} ., \mathrm{Hu}, \mathrm{C} .:$ Robust adaptive fuzzy Control for HFV with parameter uncertainty and unmodeled dynamics. IEEE Transactions on Industrial Electronics. 65(11), 8851-8860 (2018)

7. Bu, X., He, G., Wang, K.: Tracking control of airbreathing hypersonic vehicles with non-affine dynamics via improved neural back-stepping design. ISA Transactions. 75, 88-100 (2018)

8. Xu, B., Wang, D., Sun, F., Shi, Z.: Direct neural discrete control of hypersonic flight vehicle. Nonlinear Dynamics. 70(1), 269-278 (2012)

9. Wang, J., Wu, H.: Exponentially stabilizing fuzzy controller design for a nonlinear ODE-beam cascaded system and its application to flexible air-breathing hypersonic vehicle. Fuzzy Sets \& Systems. (2019) doi: 10.1016/j.fss.2019.02.023

10. Bu, X., Wu, X., Zhu, F., Huang, J., Ma, Z., Zhang, R.: Novel prescribed performance neural control of a flexible air-breathing hypersonic vehicle with unknown initial errors. ISA Transactions. 59, 149-159 (2015)

11. Bu, X., Xiao, Y., Wang, K.: A prescribed performance control approach guaranteeing small overshoot for airbreathing hypersonic vehicles via neural approximation. Aerospace Science and Technology. 71, 485-498 (2017) 
12. Bu, X., Wu, X., Huang, J., Wei, D.: A guaranteed transient performance-based adaptive neural control scheme with low-complexity computation for flexible air-breathing hypersonic vehicles. Nonlinear Dynamics. 84(4), 1-20 (2016)

13. Sun, H., Li, S., Sun, C.: Finite time integral sliding mode control of hypersonic vehicles. Nonlinear Dynamics. 73(1-2), 229-244 (2013)

14. Sun, J., Yi, J., Pu, Z., Liu, Z.: Adaptive fuzzy nonsmooth backstepping output-feedback control for hypersonic vehicles with finite-time convergence. IEEE Transactions on Fuzzy Systems. 28(10), 2320-2334 (2019)

15. Dong, C., Liu, Y., Wang, Q.: Barrier Lyapunov function based adaptive finite-time control for hypersonic flight vehicles with state constraints. ISA Transactions. 96, 163$176(2020)$

16. Basin, M., Yu, P., Shtessel, Y.: Hypersonic Missile Adaptive Sliding Mode Control Using Finite- and Fixed-Time Observers. IEEE Transactions on Industrial Electronics. 65(1), 930-941 (2017)

17. Chen, M., Wang, H., Liu, X.: Adaptive fuzzy practical fixed-time tracking control of nonlinear systems. IEEE Transactions on Fuzzy Systems. (2019) doi: 10.1109/TFUZZ.2019.2959972

18. Yu, X., Li, P., Zhang, Y.: Fixed-Time Actuator Fault Accommodation Applied to Hypersonic Gliding Vehicles. IEEE Transactions on Automation science and Engineering. (2020) doi:10.1109/TASE.2020.3008846

19. Sun, J., Yi, J., Pu, Z.: Augmented fixed-time observerbased continuous robust control for hypersonic vehicles with measurement noises. IET Control Theory \& Applications. 13(3), 422-433 (2019)

20. Jiang, B., Hu, Q., Friswell, M.: Fixed-time attitude control for rigid spacecraft with actuator saturation and faults. IEEE Transactions on Control Systems Technology. 24(5), 1892-1898 (2016)

21. Li, Y., Li, K., Tong, S.: Finite-time adaptive fuzzy output feedback dynamic surface control for MIMO nonstrict feedback systems. IEEE Transactions on Fuzzy Systems. 27(1), 96-110 (2019)

22. Li, Y., Shao, X., Tong, S.: Adaptive fuzzy prescribed performance control of non-triangular structure nonlinear systems. IEEE Transactions on Fuzzy Systems. (2019) doi:10.1109/tfuzz.2019.2937046

23. Lv, M., Baldi, S., Liu, Z.: The non-smoothness problem in disturbance observer design: a set-invariance-based adaptive fuzzy control method. IEEE Transactions on Fuzzy Systems. 27(3), 598-604 (2019)

24. Lv, M., Schutter, B., Yu, W., Zhang, W., Baldi, S.: Nonlinear systems with uncertain periodically disturbed control gain functions: adaptive fuzzy control with invariance properties. IEEE Transactions on Fuzzy Systems. 28(4), 746-757 (2020)

25. Xu, B., Guo, Y., Yuan, Y., Fan, Y., Wang, D.: Faulttolerant control using command-filtered adaptive backstepping technique: Application to hypersonic longitudinal flight dynamics. International Journal of Adaptive Control \&s Signal Processing. 30(4), 553-577 (2016)

26. Xu, B., Shi, Z., Sun, F., He, W.: Barrier lyapunov function based learning control of hypersonic flight vehicle with AOA constraint and actuator faults. IEEE Transactions on Cybernetics. 49(3), 1047-1057 (2019)

27. Li, Y.: Finite time command filtered adaptive fault tolerant control for a class of uncertain nonlinear systems. Automatica. 106(4), 117-123 (2019)

28. Li, Y., Yang, G.: Adaptive asymptotic tracking control of uncertain nonlinear systems with input quantization and actuator faults. Automatica. 72, 177-185 (2016)
29. Sun, H., Li, S., Sun, C.: Adaptive fault-tolerant controller design for airbreathing hypersonic vehicle with input saturation. Journal of Systems Engineering and Electronics. 24(3), 152-163 (2013)

30. Benchlioulis, C., Rovithakis, G.: Adaptive control with guaranteed transient and steady state tracking error bounds for strict feedback systems. Automatica. 45(2), 532-538 (2009)

31. Parker, J., Serrani, A., Yurkovich, S., Bolender, M., Doman, D.: Control-oriented modeling of an air-breathing hypersonic vehicle. Journal of Guidance Control and Dynamics. 30(3), 856-869 (2007)

32. Xu, B., Wang, D., Zhang, Y., Shi, Z.: DOB-based neural control of flexible hypersonic flight vehicle considering wind effects. IEEE Transactions on Industrial Electronics. 64(11), 8676-8685 (2017)

33. Xu, B., Shi, Z., Sun, F., He, W.: Barrier lyapunov function based learning control of hypersonic flight vehicle with AOA constraint and actuator faults. IEEE Transactions on Cybernetics. 49(3), 1047-1057 (2019)

34. Fiorentini, L., Serrani, A., Bolender, M., Doman, D.: Nonlinear robust adaptive control of flexible airbreathing hypersonic vehicles. Journal of Guidance Control \& Dynamics. 32(2), 402-417 (2009)

35. Yang, L., Liu, X., Jing, Y.: Adaptive neural networks finite-time tracking control for non-strict feedback systems via prescribed performance. Information Sciences. 468, 29-46 (2018)

36. Liu, Z., Dong, X., Xie, W., Chen, Y., Li, H.: Adaptive fuzzy control for pure-feedback nonlinear systems with non-affine functions being semi-bounded and indifferentiable. IEEE Transactions on Fuzzy Systems. 26(2), 395-408 (2018)

37. Wang, C., Zuo, Z.: Adaptive trajectory tracking control of output constrained multi-rotors systems. IET Control Theory and Applications. 8(13), 1163-1174 (2014)

38. Wang, X., Guo, J., Tang, S., Qi, S.: Fixed-time disturbance observer based fixed-time back-stepping control for an air-breathing hypersonic vehicle. ISA Transactions. 88, 233-245 (2019) 

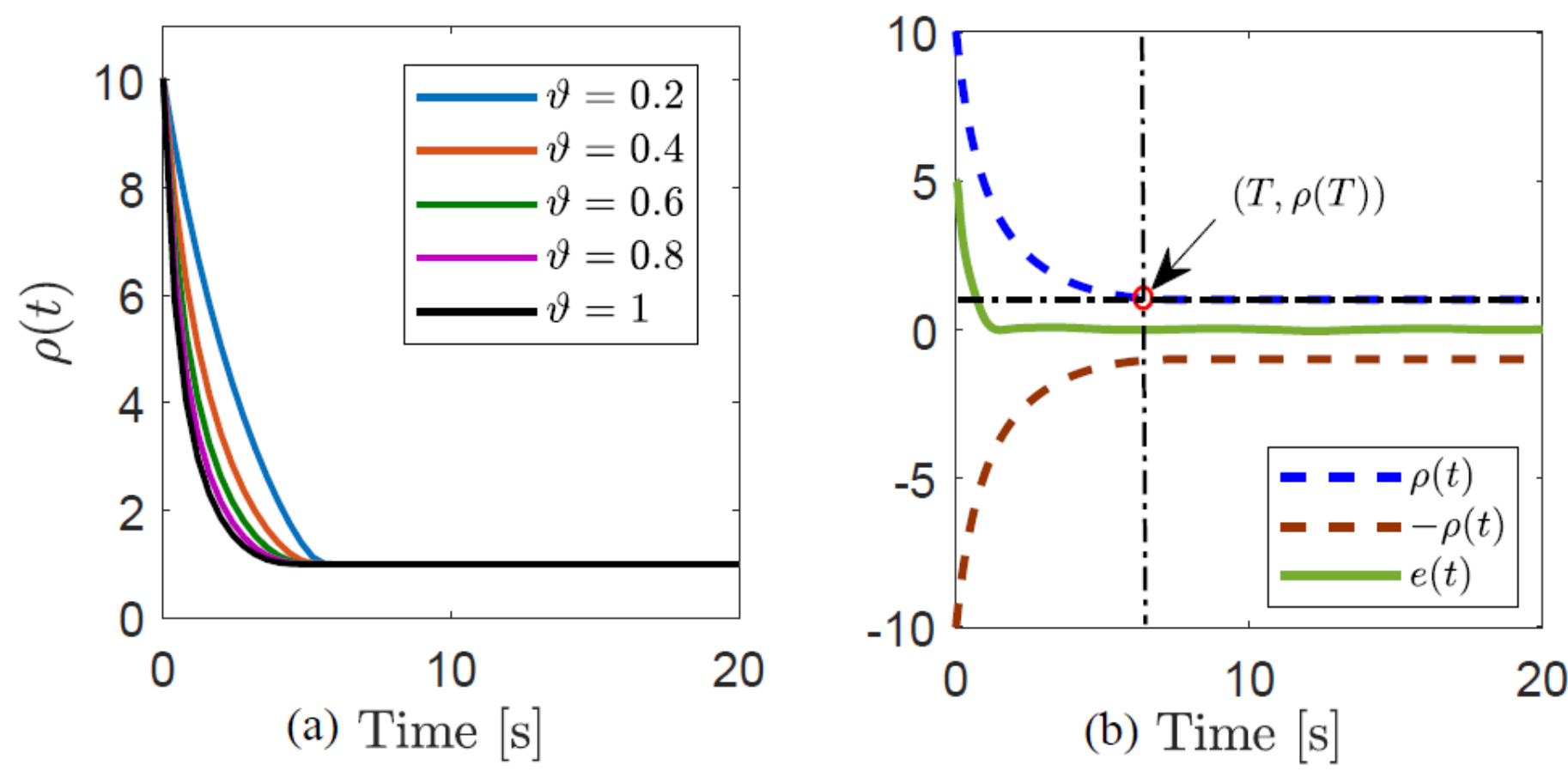

Figure 1

The convergence performance of the proposed FTPF

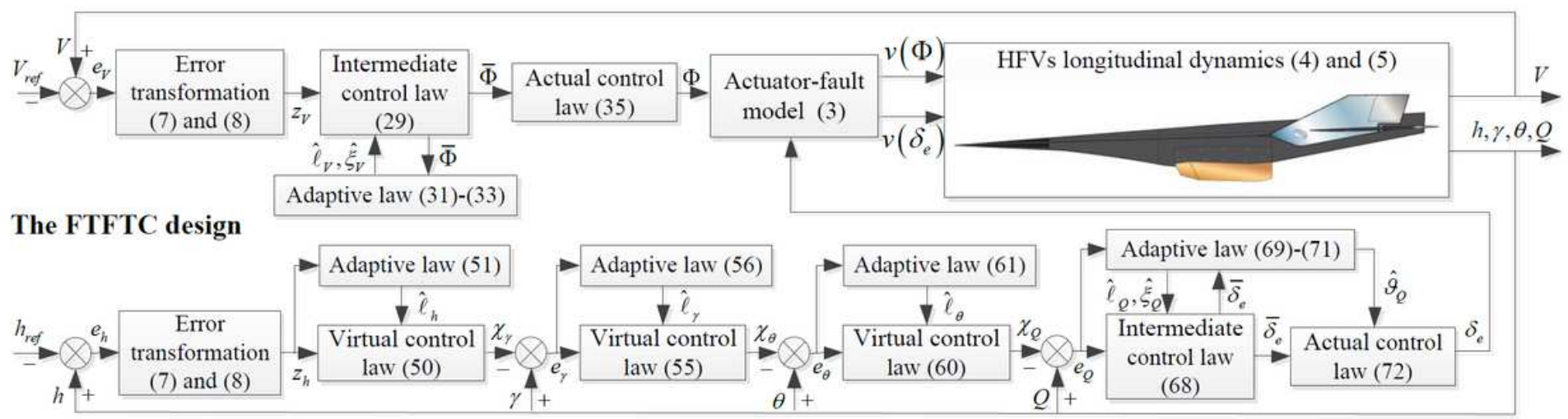

Figure 2

Overall fixed-time tolerant-control scheme for HFVs 

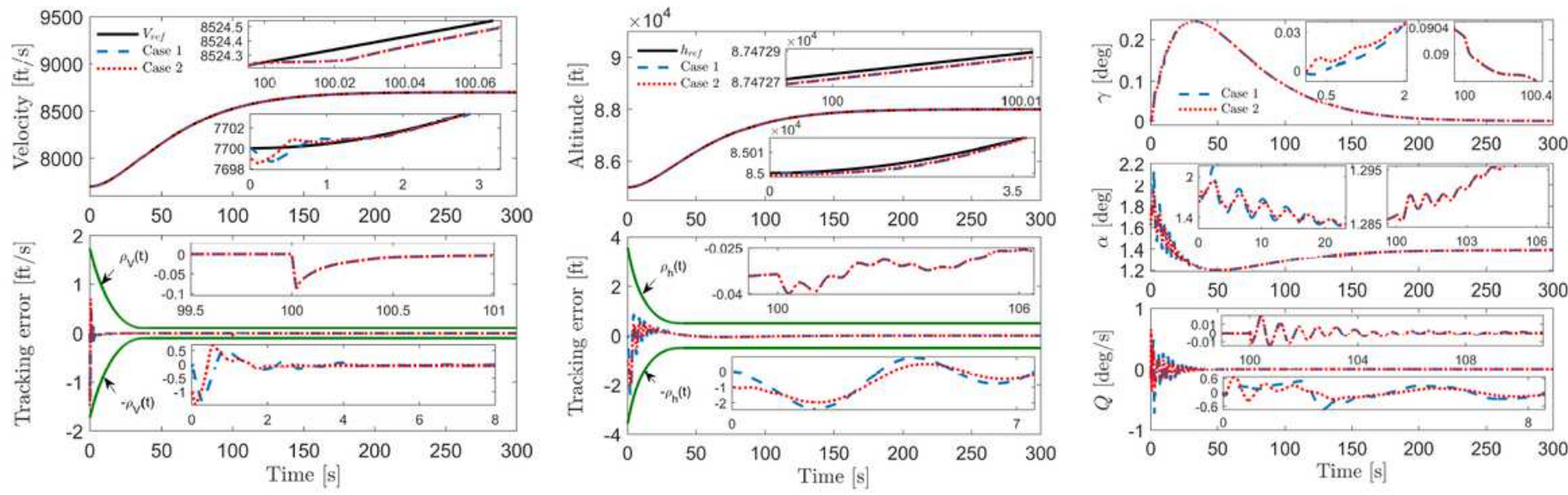

(a) Velocity tracking performance

(b) Altitude tracking performance

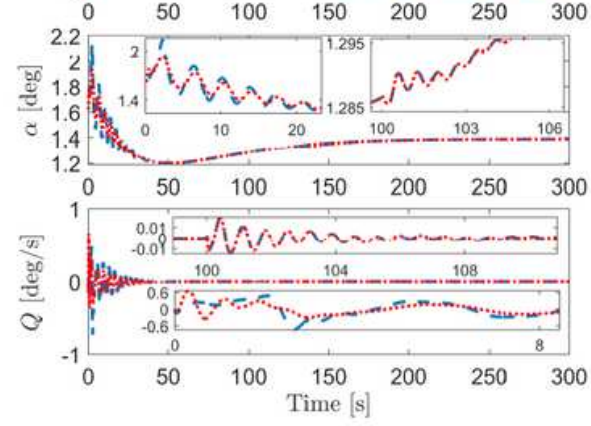

(c) Attitude angles

\section{Figure 3}

The velocity, altitude tracking performance and attitude angles under different initial states

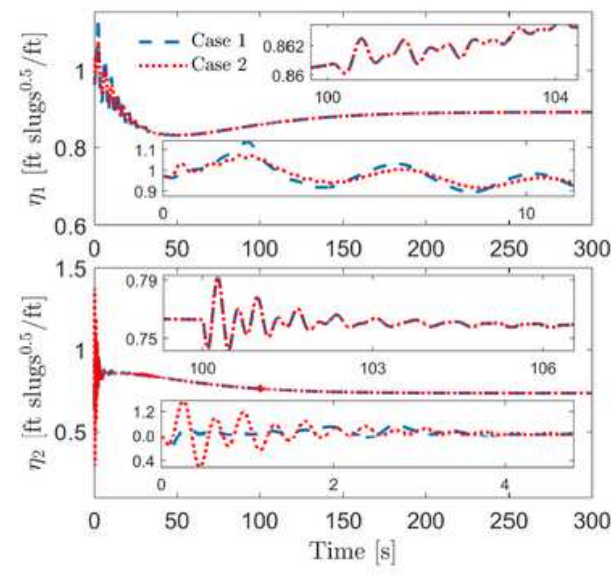

(a) Flexible states

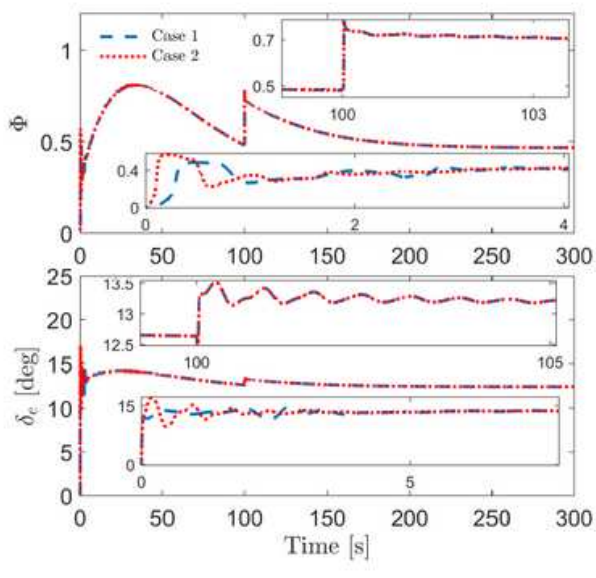

(b) Control inputs
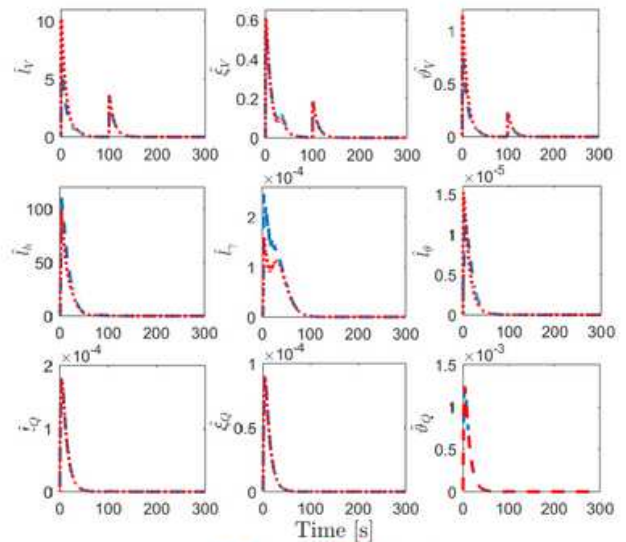

- - Case 1 - Case 2

(c) Adaptive parameters

\section{Figure 4}

The flexible states, control inputs and adaptive parameters under different initial states 

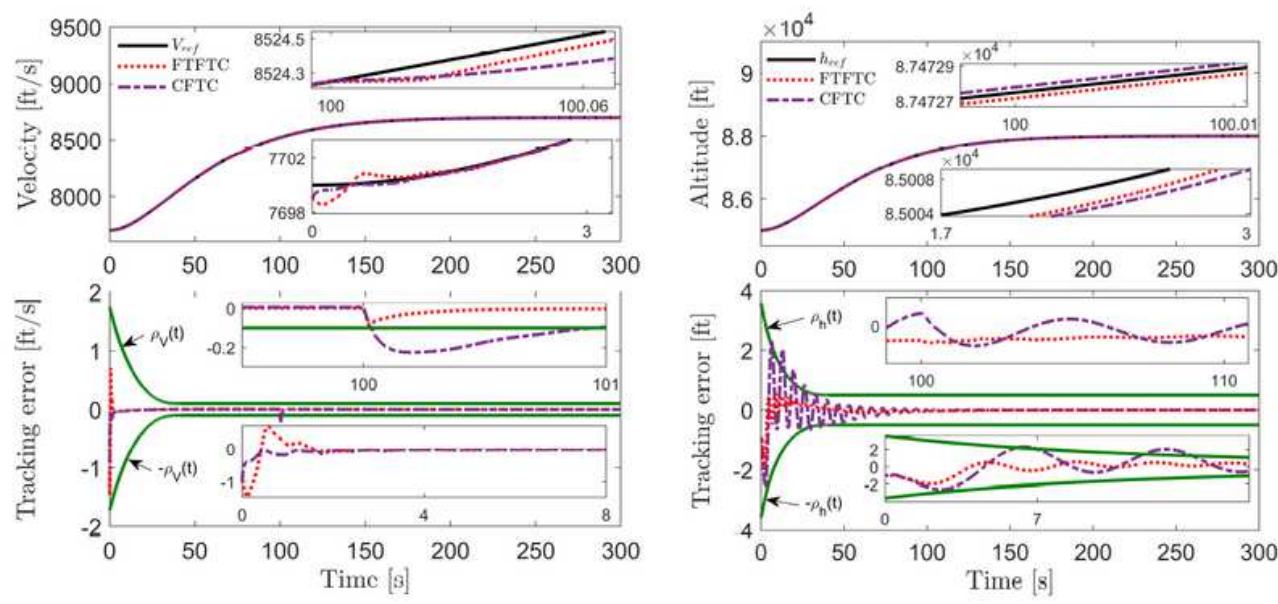

(a) Velocity tracking performance

(b) Altitude tracking performance

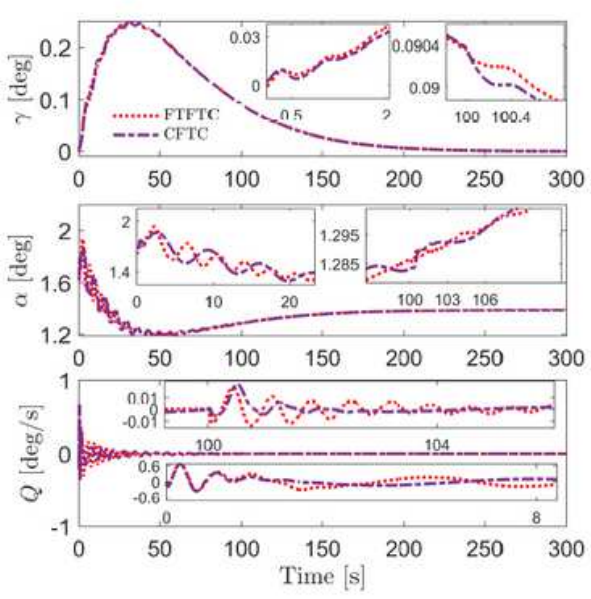

(c) Attitude angles

\section{Figure 5}

The velocity, altitude tracking performance and attitude angles via different methodologies

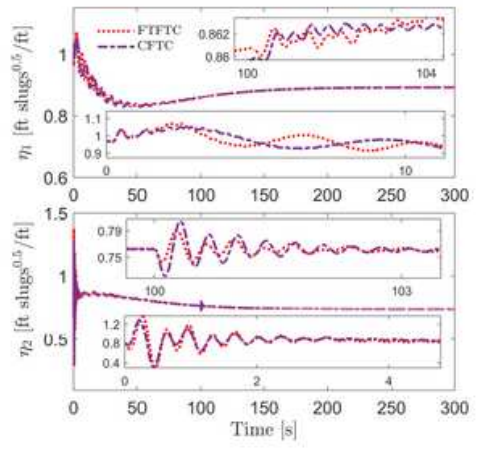

(a) Flexible states

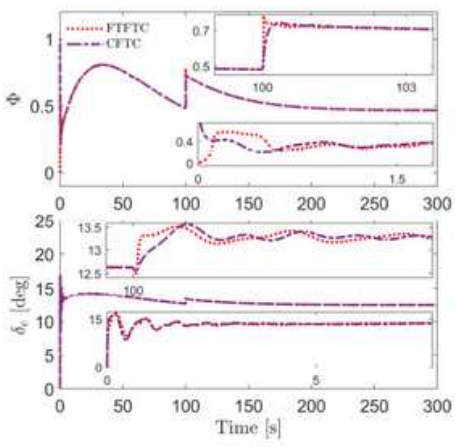

(b) Control inputs

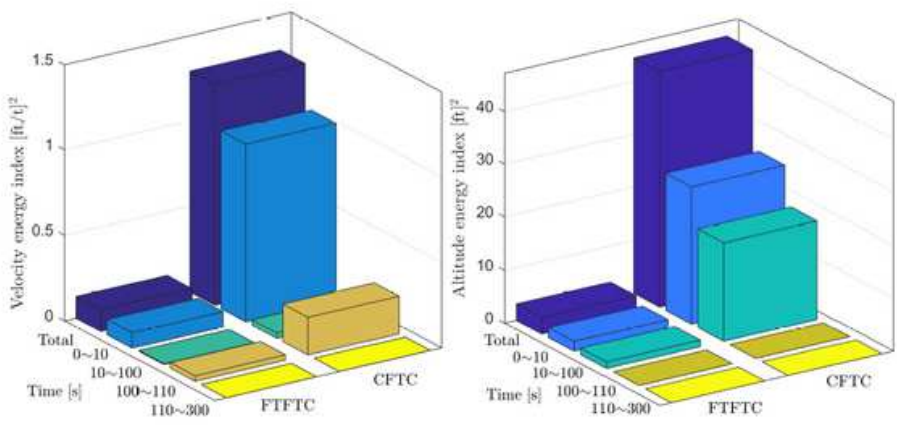

(c) Performance indexes

\section{Figure 6}

The flexible states, control inputs and performance indexes via different methodologies 